\title{
Thermodynamics of Statistical Anyons
}

\author{
Nathan M. Myers $\odot^{1, *}$ and Sebastian Deffner® ${ }^{1,2, \dagger}$ \\ ${ }^{1}$ Department of Physics, University of Maryland Baltimore County, Baltimore, Maryland 21250, USA \\ ${ }^{2}$ Instituto de Física 'Gleb Wataghin', Universidade Estadual de Campinas, Campinas, São Paulo 13083-859, \\ Brazil
}

(Received 10 February 2021; revised 26 July 2021; accepted 7 September 2021; published 19 October 2021)

\begin{abstract}
In low-dimensional systems, indistinguishable particles can display statistics that interpolate between bosons and fermions. Signatures of these "anyons" have been detected in two-dimensional quasiparticle excitations of the fractional quantum Hall effect, however experimental access to these quasiparticles remains limited. As an alternative to these "topological anyons," we propose "statistical anyons" realized through a statistical mixture of particles with bosonic and fermionic symmetry. We show that the framework of statistical anyons is equivalent to the generalized exclusion statistics (GES) pioneered by Haldane, significantly broadening the range of systems to which GES apply. We develop the full thermodynamic characterizations of these statistical anyons, including both equilibrium and nonequilibrium behavior. To develop a complete picture, we compare the performance of quantum heat engines with working mediums of statistical anyons and traditional topological anyons, demonstrating the effects of the anyonic phase in both local equilibrium and fully nonequilibrium regimes. In addition, methods of optimizing engine performance through shortcuts to adiabaticity are investigated, using both linear response and fast-forward techniques.
\end{abstract}

DOI: 10.1103/PRXQuantum.2.040312

\section{INTRODUCTION}

A unique aspect of quantum particles is that they may be truly identical - that is to say there exists no method or feature by which we would be able to distinguish one from another. The linear nature of quantum mechanics accounts for this in the form of a superposition state for the system of all possible permutations of individual particle states [1]. Such a composition is not unique, with two possible solutions distinguished by a phase of \pm 1 picked up under particle exchange. The symmetric $(+1)$ solution defines a class of fundamental particles commonly called bosons, while the antisymmetric $(-1)$ solution defines a class commonly called fermions.

This symmetrization requirement has a profound physical consequence in the form of exchange forces. A simple evaluation of the separation distance between two identical particles (see, for example, Ref. [1]) shows that bosons will tend to bunch together, while fermions will tend to

${ }^{*}$ myersn1@umbc.edu

†deffner@umbc.edu

Published by the American Physical Society under the terms of the Creative Commons Attribution 4.0 International license. Further distribution of this work must maintain attribution to the author(s) and the published article's title, journal citation, and DOI. be found farther apart. In fact, for fermions the probability of two particles being found in the exact same state is identically zero, which is the origin of the familiar Pauli exclusion principle.

The field of quantum statistics was significantly expanded when it was discovered by Leinaas and Myrheim that the differences in topology in one- and two-dimensional systems allows for the existence of a continuum of fractional statistics solutions, represented by a general phase factor of $e^{i \pi \nu}$ [2],

$$
\Psi\left(\mathbf{r}_{1}, \mathbf{r}_{2}\right)=e^{i \pi v} \Psi\left(\mathbf{r}_{2}, \mathbf{r}_{1}\right) .
$$

For $v=2 n$, where $n=0,1,2, \ldots$, the bosonic case is recovered, and for $v=2 n+1$ we obtain the fermionic case. Shortly thereafter Wilczek proposed a realization of these "any"-ons in the form of a two-dimensional quasiparticle made up of a charged particle orbiting a magnetic flux tube [3]. The name anyons signifies that interchange of particles (accomplished by successive half-rotations of each quasiparticle around the other) can produce an arbitrary phase between that of fermions and bosons as a consequence of the Aharonov-Bohm effect [3].

The study of anyons was brought into the forefront when it was proven by Arovas, Schrieffer, and Wilczek that quasiparticles entering the fractional quantum Hall effect possess not only fractional charge, but also obey fractional statistics [4]. Interest in anyons received another 
boost when it was found that non-Abelian anyons [5] could be used as key components in the development of a fault-tolerant quantum computer. For non-Abelian anyons, exchange ("braiding") does not just introduce a complex phase, but acts as a unitary transformation on the state. In this manner, combinations of braids can act as quantum logic gates [6]. As small local perturbations do not change the braiding properties of the anyons, this method of quantum computation is very robust against noise [6]. Experimental access to non-Abelian anyons remains a challenge, however indirect evidence of non-Abelian anyonic states has been found [7-9]. See Ref. [10] for a more detailed review on topological quantum computation and the role of anyons. While non-Abelian anyons are necessary for implementing topological quantum computation, understanding the properties of Abelian anyons can provide important insight into the general behavior of anyons that may be useful in controlling their non-Abelian counterparts.

Discussion of anyons in one dimension requires some additional subtlety, as exchange by rotation is no longer valid. In this context, Haldane introduced a dimensionindependent approach to anyonic statistics, generalized exclusion statistics (GES), based on a generalization of the Pauli exclusion principle [11]. In Haldane's approach, particle nature is quantified by a statistical interaction that determines the degree to which two identical particles can occupy the same state [11]. Notably, this approach classifies particle type solely on degree of repulsion and as such identifies "hardcore-bosons" and fermions as equivalent, despite differences in low-energy behavior [11]. While closely linked to Wilczek's formulation of anyons, Haldane's GES anyons are distinctly different, as their behavior is based on generalizing the exclusion principle, rather than the exchange behavior [12].

Haldane's work was expanded upon by $\mathrm{Wu}$, who derived the statistical distribution for an ideal anyon gas [13]. This approach continued to receive attention when it was shown that the Calogero-Sutherland model gas, consisting of bosons or fermions in a harmonic potential with an inverse square-law interaction, could be treated as a system of noninteracting anyons obeying Haldane's generalized exclusion statistics [14-19]. The same was found to be true for a one-dimensional (1D) Lieb-Liniger [20] model gas of interacting bosons, whose thermodynamics were first studied by Yang and Yang [21,22], with the distinction that the statistical interaction occurs between particles of different momenta [23-26]. Notably, in the case of the Lieb-Liniger model, the parameter controlling the statistics cannot be directly identified with the coupling constant of the interaction, as in the Calogero-Sutherland model, except in the bosonic and fermionic limits [27].

Further work demonstrated that two-dimensional (2D) trapped Bose gases with contact interactions can also be treated as ideal systems obeying GES [28,29]. GES has even been extended to "topological anyons," such as those proposed by Leinaas, Myrheim, and Wilczek [2,3], where it has been found that dimensionally confined, interacting topological anyons can be mapped to ideal topological anyons that obey GES [30-41].

The statistical mechanics of both GES and topological anyons have seen significant study. For GES anyons, much of this work has focused on analyzing the distribution function $[13,42-46]$, partition functions [14,15,18,47], the virial coefficients $[12,24,44]$, the equation of state $[13,15,24,47]$, and deriving thermodynamic behavior using the thermodynamic Bethe ansatz $[23,42,48,49]$. In the case of topological anyons, the analysis has followed similar lines, with detailed examinations of partition functions $[50,51]$ and the virial coefficients $[43,51-56]$. Topological anyon thermodynamics has also been studied in the context of relativistic systems [57] and phase transitions [58]. For the case of non-Abelian topological anyons confined to the lowest Landau level of a strong magnetic field, it has been found that the virial coefficients are independent of the statistics parameter [59].

While much focus has been placed on the statistical mechanics of anyons, significantly less has been directed towards examining the impact of anyonic statistics on traditionally studied thermodynamic systems such as heat engines. The emerging field of quantum thermodynamics [60] has also given rise to a resurgence of interest in quantum control techniques in the form of shortcuts to adiabaticity, finite-time methods for achieving a final quantum state that would normally result only from infinitely slow driving $[61,62]$. Both of these topics are directly relevant to the development of quantum devices. Heat engines provide the paradigmatic framework for studying the thermodynamics of a system in a practical context, and quantum heat engines have been used to model quantum devices ranging from masers [63] to quantum computers [64]. Shortcuts to adiabaticity have a wide range of applications from optimizing the performance of quantum engines to enhancing the speed of quantum-information processing in both gate-based and quantum-annealing paradigms [61]. As such, understanding how the thermodynamic behavior of anyons affects heat-engine performance and shortcuts to adiabaticity will help shed light on how their unique features may be applied in quantum technologies, and how future experiments may probe these features.

While the theoretical study of anyons is well established, it is only in recent years that experimental techniques have advanced to the point that detection of anyons is feasible. For the case of fractional quantum Hall state anyons, a popular detection method is based on implementing quasiparticle interferometers whose interference effects depend directly on the anyonic phase [65-68]. Numerous experimental attempts have followed this approach [69-74] but distinguishing signatures of the anyonic phase from interference effects arising from other factors, such 
as Coulomb blockading and Aharonov-Bohm interference, has proven elusive until recently [75].

The difficulty in accessing fractional quantum Hall state anyons has led to alternative methods of experimentally studying their properties. A promising route that we focus on in this work is motivated by the celebrated Hong-Ou-Mandel (HOM) effect first observed in photonic interferometry [76].

In Sec. II we establish an implementation of anyons from a statistical mixture of boson and fermion pairs, which we refer to as "statistical anyons." We show that statistical anyons, despite different construction, behave equivalently to Haldane's generalized exclusion principle anyons. In Sec. III we examine the equilibrium thermodynamic properties of statistical and Abelian topological anyons, including entropy, heat capacity, and free energy. In Sec. IV we compare the performance of an endoreversible quantum Otto engine with a working medium of statistical anyons to one with a working medium of topological anyons. In Sec. V, we extend our analysis of the statistical anyon engine to the fully nonequilibrium regime. In Sec. VI we explore the role of anyonic statistics in the implementation of shortcuts to adiabaticity, which can be used to enhance thermal machine performance by increasing power without loss of efficiency. In Sec. VII we conclude with a perspective on future research directions.

\section{II. "STATISTICAL" ANYONS}

In the typical HOM effect a pair of entangled photons are incident symmetrically on a two-port 50:50 beam splitter. Assuming all other degrees of freedom of the photons are identical (such as frequency and polarization), the initial bosonic spatial state is described by a symmetric superposition of each input,

$$
\left|\psi_{i}^{B}\right\rangle=\frac{1}{\sqrt{2}}\left(|a\rangle_{1}|b\rangle_{2}+|b\rangle_{1}|a\rangle_{2}\right) .
$$

The operation of the beam splitter evolves state $|a\rangle \rightarrow$ $(1 / \sqrt{2})(|c\rangle+i|d\rangle)$ and state $|b\rangle \rightarrow(1 / \sqrt{2})(i|c\rangle+|d\rangle)$ with the imaginary component denoting the phase shift of $\pi$ picked up upon reflection. Carrying out this evolution on each input state while keeping track of the particle indices we find that the only states to survive are those in which both photons exit the same beam-splitter port,

$$
\left|\psi_{f}^{B}\right\rangle=\frac{i}{\sqrt{2}}\left(|c\rangle_{1}|c\rangle_{2}+|d\rangle_{1}|d\rangle_{2}\right) .
$$

Physically, this is a manifestation of the effective attraction between bosons (typically this is referred to as "boson bunching") [76].

The HOM effect can be extended to cases in which the other degrees of freedom of the photons are not identical [77]. Consider a case in which the photons are prepared in a Bell state basis in polarization. The four possible Bell pairs are

$$
\begin{aligned}
& \left|\Phi_{A}\right\rangle=\frac{1}{\sqrt{2}}(|00\rangle+|11\rangle), \\
& \left|\Phi_{B}\right\rangle=\frac{1}{\sqrt{2}}(|00\rangle-|11\rangle), \\
& \left|\Phi_{C}\right\rangle=\frac{1}{\sqrt{2}}(|01\rangle+|10\rangle), \\
& \left|\Phi_{D}\right\rangle=\frac{1}{\sqrt{2}}(|01\rangle-|10\rangle),
\end{aligned}
$$

where here $|0\rangle$ and $|1\rangle$ represent orthogonal polarization states. We can use this additional degree of freedom to encode fermionic, and ultimately anyonic, statistics into the behavior of the photons [78-81]. In this manner the photons can be thought of as a "quantum substrate" on which we construct our desired statistics.

We note that $\left|\Phi_{A}\right\rangle,\left|\Phi_{B}\right\rangle$, and $\left|\Phi_{C}\right\rangle$ are symmetric under exchange, while $\left|\Phi_{D}\right\rangle$ is antisymmetric. As photons are bosons, their overall wave function must still be symmetric. This can occur in one of two ways: (i) a symmetric spatial state paired with one of the three symmetric polarization states,

$$
\left|\Psi_{i}^{B}\right\rangle=\frac{1}{\sqrt{2}}\left(|a\rangle_{1}|b\rangle_{2}+|b\rangle_{1}|a\rangle_{2}\right) \otimes\left|\Phi_{j}\right\rangle,
$$

where $j=\{A, B, C\}$, or (ii) an antisymmetric spatial state paired with the antisymmetric polarization state,

$$
\left|\Psi_{i}^{F}\right\rangle=\frac{1}{\sqrt{2}}\left(|a\rangle_{1}|b\rangle_{2}-|b\rangle_{1}|a\rangle_{2}\right) \otimes\left|\Phi_{D}\right\rangle,
$$

Assuming it is nonpolarizing, the beam splitter acts only on the spatial portion of the wave function. For case (ii), as shown in Eq. (6), the output spatial state is

$$
\left|\psi_{f}^{F}\right\rangle=\frac{1}{\sqrt{2}}\left(|c\rangle_{1}|d\rangle_{2}-|d\rangle_{1}|c\rangle_{2}\right)
$$

guaranteeing that both photons exit opposite ports of the beam splitter. This is a result of the effective repulsion between fermions, and can be seen as a manifestation of the Pauli exclusion principle. Despite the fact that photons are fundamentally bosons, since the beam splitter accesses only the antisymmetric portion of the overall state, they behave exactly as fermions. Figure 1(a) summarizes the possible outcomes for the two-photon input states. Notably, both bunching and antibunching behavior vanishes if the photons become distinguishable (say by increasing the flight time for just one port of the beam splitter) [77]. 
(a)

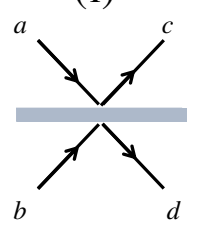

(iii)

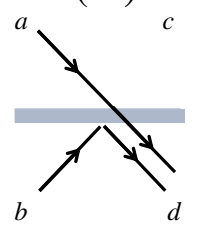

(ii)

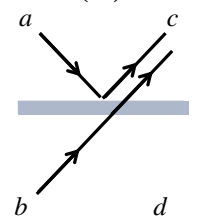

(iv)

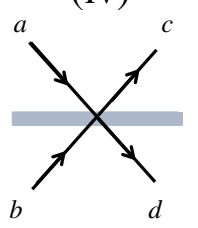

(b)
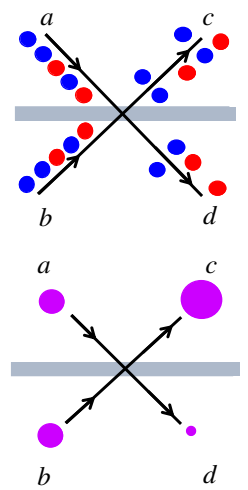

FIG. 1. (a) All possible photon transmission and reflection combinations. For symmetric (bosonic) states possibilities (i) and (iv) interfere deconstructively while for antisymmetric (fermionic) states they interfere constructively. (b) Representation of statistical anyons for $N=5$. Top figure: $N$ particle pairs are incident, with blue particles representing pairs with symmetric entanglement and red pairs with antisymmetric entanglement. Bottom figure: The equivalent statistical anyonic representation for each particle pair, with particle size representing the probability density of finding a particle at that output port.

This effect can be extended to anyonic statistics by introducing a phase that tunes between the symmetric and antisymmetric Bell states. Phase control can be achieved using a rotated polarization beam splitter [81], adjusting path length [78,79], or introducing phase plates [80], making this method of simulating anyonic behavior very experimentally accessible. Here we propose, to the best of our knowledge, a new and straightforward method of introducing this anyonic phase through a statistical mixture of symmetrically and antisymmetrically entangled photon pairs. Consider an incident anyonic wave function of the form,

$$
\left|\psi_{i}^{A}\right\rangle=\frac{1}{\sqrt{2}}\left(|a\rangle_{1}|b\rangle_{2}+e^{i \pi v}|b\rangle_{1}|a\rangle_{2}\right)
$$

Applying the beam splitter unitary to Eq. (8), we see after a few lines of algebra that the probability of both photons exiting the same port is

$$
P_{\text {same }}=\frac{1}{2}[1+\cos (\pi \nu)] .
$$

As $v$ is varied, we see that $P_{\text {same }}$ interpolates between zero and one. If we consider $N$ photon pairs incident on the beam splitter, of which some fraction are symmetrically entangled and the rest antisymmetrically entangled, we realize that the same behavior can be replicated on average by simply changing the distribution of symmetrically and antisymmetrically entangled pairs. In this framework $v$ is a measure of the probability that any given pair in the mixture is symmetrically entangled, $v=v\left(p_{B}\right)$. This behavior is shown pictorial in Fig. 1(b).

We refer to this implementation as "statistical anyons" in contrast to "topological anyons," that appear in Wilczek's charge and flux tube realization [3], or in quasiparticle excitations in the fractional quantum Hall regime [4]. While both formulations pick up an anyonic phase under particle exchange and display behavior interpolating between that of fermions and bosons, in the present statistical anyon framework this is a result of averaging over the behavior of a large number of particles, while for topological anyons it is a property of the quasiparticles themselves. Note that when we refer to topological anyons, we are considering specifically Abelian particles, whose exchange introduces a phase a $e^{i \pi v}$ [56].

From a topological standpoint, there is another important distinction between statistical and topological anyons. It is well established that the configuration space of topological anyon statistics is a representation of the braid group, rather than the permutation group, as is the case for bosonic and fermionic statistics [2,56]. This difference arises as in two dimensions, two repeated exchanges is a topologically distinct operation from doing nothing, while in three dimensions these operations are topologically equivalent $[2,56]$. This leads to the physical consequence that the direction of the exchange is significant for topological anyons, as two exchanges in the same direction will result in a wave function different from the original by a phase of $e^{2 i \pi v}$.

Since statistical anyons are constructed from an average over a mixture of bosons and fermions, two repeated exchanges will always return the original wave function, independent of exchange direction. The average phase for any pair of particles in the mixture is then given by the average over the bosonic and fermionic phase factors and as such will always fall between -1 and 1 . Thus the statistical anyon mixture corresponding to the topological anyon phase of $e^{i \pi v}$ will be weighted such that the average phase of the mixture is $\cos \left[\pi \nu\left(p_{B}\right)\right]$. We can represent the two-dimensional space of possible topological anyon phases as a circle, with bosonic and fermionic phases given by diametrically opposite points. The space of possible averaged statistical anyon phases is then represented by the diameter line of the circle. In this geometric picture the averaged statistical anyon phase is the projection of the corresponding topological anyon phase onto the real axis. These differences between topological and statistical anyons are illustrated graphically in Fig. 2.

We see from this comparison that, as a representation of the braid group, topological anyons show increased complexity in comparison to statistical anyons. This leads to the motivating questions of this work: How does the thermodynamics of topological and statistical anyons compare? Can the simpler, more experimentally accessible 
(a)

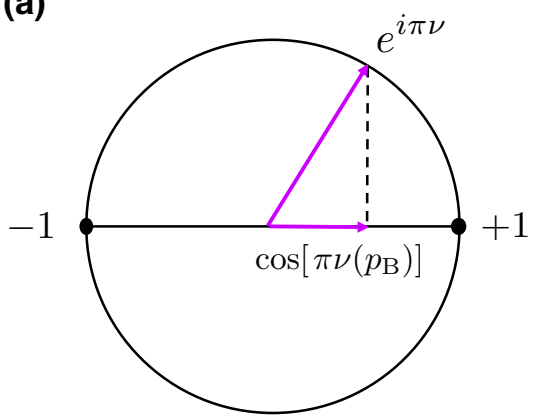

(b)
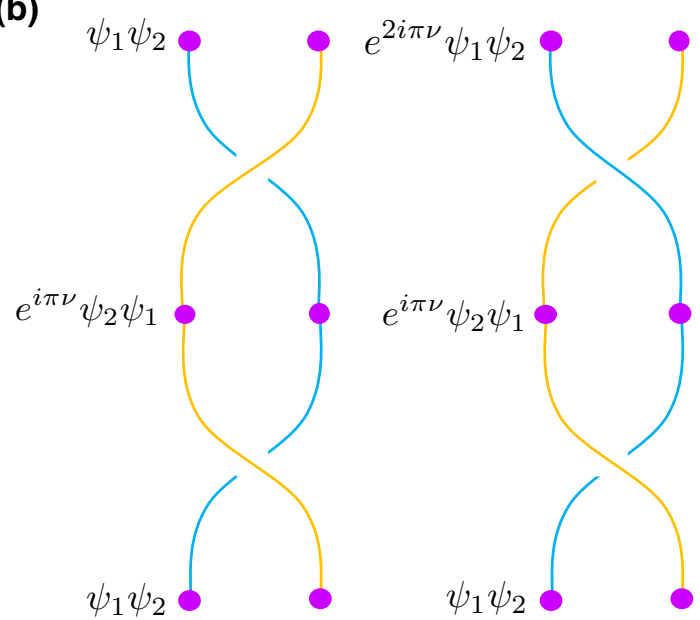

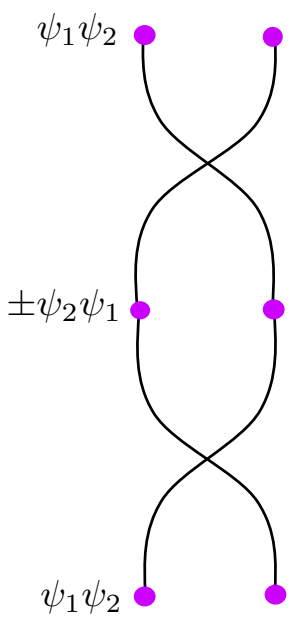

FIG. 2. (a) Comparison between the exchange phases of topological and statistical anyons. For topological anyons the direction of the exchange (clockwise or counterclockwise) determines the sign of the anyonic phase. As such, the space of possible topological anyon exchange phases can be represented by a circle, with bosonic and fermionic symmetry lying on opposite sides. For statistical anyons, the anyonic phase is an average over $N_{B}$ bosonic phase factors and $N_{F}$ fermionic phase factors. As such, the space of possible statistical anyon phases is confined to a line between -1 and +1 . (b) Comparison between topological and statistical anyons undergoing two interchanges. For topological anyons two exchanges in opposite directions leave the wave function unchanged (left diagram), while two exchanges in the same direction results in the original wave function multiplied by an additional phase of $e^{2 i \pi v}$ (middle diagram). For statistical anyons the direction of the exchange is irrelevant, with two exchanges always resulting in an unchanged wave function (right diagram).

statistical anyons replicate any of the unique properties of topological anyons? Do statistical anyons themselves display intricate thermodynamic behavior that can be exploited?

\section{A. Statistical anyon wave function}

To better understand the relationship between statistical and topological anyons, let us consider extending the notion of statistical anyons beyond the realm of quantum optics. For a single pair of bosonic particles the spatial wave function is given by

$\Psi_{B}(\mathbf{x})=\frac{1}{\sqrt{2\left(1+\delta_{n_{1}, n_{2}}\right)}}\left[\psi_{n_{1}}(x) \psi_{n_{2}}(y)+\psi_{n_{1}}(y) \psi_{n_{2}}(x)\right]$,

where $\psi_{n}(x)$ is the normalized single-particle eigenstate corresponding to quantum number $n$ and $\mathbf{x}=(x, y)$. Note that the normalization coefficient for bosons changes when both particles occupy the same state, as in this case the unnormalized wave function becomes identical to the wave function of two distinguishable particles with an additional factor of 2. Similarly, the wave function of a fermionic particle pair is

$$
\Psi_{F}(\mathbf{x})=\frac{1}{\sqrt{2}}\left[\psi_{n_{1}}(x) \psi_{n_{2}}(y)-\psi_{n_{1}}(y) \psi_{n_{2}}(x)\right] .
$$

Here we require no $\delta$ function in the normalization, as two fermions will never occupy the same state. The total wave function for $N$ independent particle pairs, of which $N_{B}$ of the pairs are symmetric under exchange and $N_{F}=N-N_{B}$ of the pairs are antisymmetric under exchange is then

$$
\Psi\left(\mathbf{x}_{1}, \mathbf{x}_{2}, \ldots, \mathbf{x}_{N}\right)=\prod_{j=1}^{N_{B}} \Psi_{B}\left(\mathbf{x}_{j}\right) \prod_{k=N_{B}+1}^{N} \Psi_{F}\left(\mathbf{x}_{k}\right) .
$$

This wave function can be equivalently represented in the framework of statistical anyons as

$$
\Psi\left(\mathbf{x}_{1}, \mathbf{x}_{2}, \ldots, \mathbf{x}_{N}\right)=\prod_{j=1}^{N} \Psi_{A}\left(\mathbf{x}_{j}\right),
$$

where

$$
\begin{aligned}
\Psi_{A}\left(\mathbf{x}_{j}\right)= & \frac{1}{\sqrt{2\left(1+\delta_{n_{1}, n_{2}}\right)}}\left[\psi_{n_{1}}\left(x_{j}\right) \psi_{n_{2}}\left(y_{j}\right)\right. \\
& \left.+e^{i \pi v_{j}} \psi_{n_{1}}\left(y_{j}\right) \psi_{n_{2}}\left(x_{j}\right)\right] .
\end{aligned}
$$

We pause here to note a significant difference between statistical and topological anyons. As we see in Eqs. (13) and (14), the statistical anyon wave function can always be constructed from a product of superpositions of the single-particle wave functions. This is not generally true for the wave function of topological anyons, outside of the bosonic and fermionic limits of the anyonic phase $[43,51,56]$. 
The product in Eq. (13) must give $N_{B}$ symmetric wave functions and $N_{F}$ antisymmetric wave functions. This condition is fulfilled if the anyonic phase in Eq. (14) is given by $v_{j}=\Theta\left(j-N_{B}-1\right)$, where $\Theta(\cdot)$ is the Heaviside step function, using the convention $\Theta(0)=1$. In this framework, the anyonic factor in Eq. (14) can be thought of as giving the "average" phase picked up under exchange for any one of the $N$ pairs of particles. For large $N$ we can express the number of bosonic and fermionic particle pairs as $N_{B}=N p_{B}$ and $N_{F}=N p_{F}=N\left(1-p_{B}\right)$ where $p_{B}\left(p_{F}\right)$ is the probability of a particle pair having bosonic (fermionic) symmetry.

In the limit of a single two-particle system, the anyonic wave function becomes

$$
\Psi_{A}(\mathbf{x})=\frac{1}{\sqrt{2\left(1+\delta_{n_{1}, n_{2}}\right)}}\left[\psi_{n_{1}}(x) \psi_{n_{2}}(y) \pm \psi_{n_{1}}(y) \psi_{n_{2}}(x)\right]
$$

with the sign determined by whether $p_{B}$ is 0 or 1 , as in reality each individual pair of particles must be either bosons or fermions.

\section{B. Statistical anyons in second quantization}

The statistical anyon framework can also be extended to second quantization, from which we can determine the appropriate commutation relations. For topological anyons, the creation and annihilation operators can be determined from the bosonic or fermionic operators via the Jordan-Wigner transformation, and obey the following commutation relations [82]:

$$
\begin{aligned}
a\left(\mathbf{x}_{\mathcal{C}}\right) a\left(\mathbf{y}_{\mathcal{C}}\right)-e^{-i \pi v} a\left(\mathbf{y}_{\mathcal{C}}\right) a\left(\mathbf{x}_{\mathcal{C}}\right) & =0 \\
a\left(\mathbf{x}_{\mathcal{C}}\right) a^{\dagger}\left(\mathbf{y}_{\mathcal{C}}\right)-e^{i \pi v} a^{\dagger}\left(\mathbf{y}_{\mathcal{C}}\right) a\left(\mathbf{x}_{\mathcal{C}}\right) & =0 \\
a^{\dagger}\left(\mathbf{x}_{\mathcal{C}}\right) a\left(\mathbf{y}_{\mathcal{C}}\right)-e^{i \pi v} a\left(\mathbf{y}_{\mathcal{C}}\right) a^{\dagger}\left(\mathbf{x}_{\mathcal{C}}\right) & =0 \\
a^{\dagger}\left(\mathbf{x}_{\mathcal{C}}\right) a^{\dagger}\left(\mathbf{y}_{\mathcal{C}}\right)-e^{-i \pi v} a^{\dagger}\left(\mathbf{y}_{\mathcal{C}}\right) a^{\dagger}\left(\mathbf{x}_{\mathcal{C}}\right) & =0 .
\end{aligned}
$$

Note that the commutation relations are dependent on the curve $\mathcal{C}$, which indicates the direction of the exchange rotation. As the topological anyon operators are a representation of the braid group, the phase picked up depends on whether or not the exchange was performed clockwise or counterclockwise (as illustrated in Fig. 2). We see that $v=0$ restores the boson commutation relations and $v=1$ restores the fermion anticommutation relations.

Notably, for particles at the same position the anyonic phase cancels out and the canonical commutation relation reduces to $[43,82]$

$$
a\left(\mathbf{x}_{\mathcal{C}}\right) a^{\dagger}\left(\mathbf{x}_{\mathcal{C}}\right) \pm a^{\dagger}\left(\mathbf{x}_{\mathcal{C}}\right) a\left(\mathbf{x}_{\mathcal{C}}\right)=1,
$$

with the $(+)$ occurring if the anyonic operators are constructed from transformed fermionic operators, and the (-) if they are constructed from transformed bosonic operators. In general, topological anyons act as "hard-core" particles for all $v$ other than $v=0$, obeying an exclusion principle [43]. In this sense, the anyonic phase parameter can be thought of as quantifying the degree of repulsion between two identical particles [43].

To determine the commutation relations for statistical anyon creation and annihilation operators, we follow a similar approach to the construction of the statistical anyon wave function in the first quantization. Let us consider $N$ Fock states. Starting each in the vacuum state, we can construct a single occupancy state from the application of the corresponding creation operator. We apply the bosonic creation operator to $N_{B}$ states and the fermionic creation operator to $N_{F}=N-N_{B}$ states,

$$
|1\rangle_{1}|1\rangle_{2} \cdots|1\rangle_{N}=\prod_{j=1}^{N_{B}} b_{j}^{\dagger}|0\rangle_{j} \prod_{k=N_{B}+1}^{N} f_{k}^{\dagger}|0\rangle_{k} .
$$

We can equivalently represent this state in the statistical anyon picture using a statistical anyon creation operator,

$$
|1\rangle_{1}|1\rangle_{2} \cdots|1\rangle_{N}=\prod_{j=1}^{N} s_{j}^{\dagger}|0\rangle_{j}
$$

The operator $s_{j}^{\dagger}$ must reduce to the bosonic creation operator for $j \leq N_{B}$ and to the fermionic creation operator for $j>N_{B}$. We can similarly define a statistical anyon annihilation operator that must reduce to the bosonic and fermionic annihilation operators under the same conditions. With these restrictions, we can construct the statistical anyon commutation relations as follows:

$$
\begin{aligned}
& s_{j}^{\dagger} s_{j}-e^{i \pi \Theta\left(j-N_{B}-1\right)} s_{j} s_{j}^{\dagger}=1, \\
& s_{j} s_{j}^{\dagger}-e^{i \pi \Theta\left(j-N_{B}-1\right)} s_{j}^{\dagger} s_{j}=1, \\
& s_{j}^{\dagger} s_{j}^{\dagger}-e^{i \pi \Theta\left(j-N_{B}-1\right)} s_{j}^{\dagger} s_{j}^{\dagger}=0, \\
& s_{j} s_{j}-e^{i \pi \Theta\left(j-N_{B}-1\right)} s_{j} s_{j}=0 .
\end{aligned}
$$

In the limit of a single particle the canonical commutation reduces to

$$
s_{j} s_{j}^{\dagger} \pm s_{j}^{\dagger} s_{j}=1
$$

as in reality the particle is either a boson or fermion. This echoes Eq. (17), where the form of the commutation relation depends on whether the anyonic operators are constructed from transformed fermionic or bosonic operators. 


\section{Statistical anyons and generalized exclusion statistics}

A notable difference between statistical and topological anyons is the lack of an exclusion principle, except in the fermionic limit. Instead, statistical anyons admit "partially occupied" states that arise from averaging over the occupancy of all $N$ systems. In this sense, the statistical anyon framework is more akin to Haldane's generalized exclusion statistics [11]. GES is constructed by extending the Pauli exclusion principle through the definition of a parameterized differential relation that quantifies the change in the dimension of the Hilbert space of a discrete-state system upon a change in the particle number [11],

$$
\Delta d_{\mathrm{GES}}=-g \Delta N .
$$

For bosons, with infinite possible state occupancy, the dimension is independent of the particle number, thus $g=$ 0 . For fermions, subject to the full exclusion principle, the dimension scales directly with each additional particle, thus $g=1$. GES statistics anyons have been shown to manifest in confined, interacting gasses, such as the Calogero-Sutherland model gas $[14,16,17,19]$. Notably, this implementation of GES anyons can be directly mapped to topological anyons confined to the lowest Landau level [13,15,47,83-85]. More recently, it has been shown that, in general, the thermodynamic potential for a gas of particles with a discrete one-body spectrum obeying GES can be constructed from a generalization of the relation found for Calogero model GES anyons or lowest Landau level topological anyons [47].

Other physical systems shown to host GES anyons include Lieb-Liniger and hard-core Tonks-Girardeau gasses with $\delta$-function potentials $[30,31,33-41,86]$ as well as Hubbard chains $[87,88]$, whose anyonic behavior can be imitated in ultracold gasses [89]. More generally, it has been shown that there exists a class of integrable models solvable by the thermodynamic Bethe ansatz can be recast in terms of ideal quasiparticles obeying GES $[16,23,42,48,49]$.

In the statistical anyon framework, the change in the Hilbert-space dimension will be given by the sum of the dimension change if the particles are bosons and the dimension change if they are fermions, weighted by the respective probabilities,

$$
\Delta d_{\mathrm{SA}}=p_{B} \Delta d_{B}+p_{F} \Delta d_{F} .
$$

Noting $\Delta d_{B}=0$ and $\Delta d_{F}=-\Delta N$, this simplifies to

$$
\Delta d_{\mathrm{SA}}=-p_{F} \Delta N .
$$

Comparing Eq. (24) to Eq. (22) we see that the parameter $g$ in the GES framework is identical to the fermion probability in the statistical anyon framework. This demonstrates that the statistical anyon framework is fully equivalent to GES anyons.

The first indications that GES anyons could be described in terms of bosons and fermions came when Murthy and Shankar [14] noted that a Calogero-Sutherland model gas could be described by ideal GES anyons, and that the resulting partition function could be factored into bosonic and fermionic components raised to the power of the GES parameter $g$ and $g-1$, respectively. Here we have demonstrated explicitly that GES can be constructed from the average behavior of an ideal statistical mixture of bosons and fermions, and identified the GES parameter with the statistical weights of the mixture.

For bookkeeping ease, we conclude this section by comparing the features of topological, statistical, and GES anyons in Table I.

\section{EQUILIBRIUM THERMODYNAMICS OF ANYONS}

\section{A. 1D statistical anyons}

The first step in understanding the thermodynamics of anyonic systems is to examine how the thermodynamic quantities such as internal energy, entropy, and heat capacity depend on the anyonic phase. To determine this, we need the proper partition function for our system. Motivated by the manifestation of GES in trapped, interacting gasses, let us consider two statistical anyons in a 1D harmonic potential. However, in this case we consider the particles to be noninteracting. Trapped boson-fermion mixtures have seen previous study both theoretically [ 90 92] and experimentally [93-95], but this work has focused primarily on effects on the ground-state configurations that arise from interactions between the boson and fermion species. In the statistical anyon framework we instead consider the average collective behavior that arises in the ideal gas limit of such a mixture.

TABLE I. Comparison of the features and behavior of topological, statistical, and GES anyons.

\begin{tabular}{lcccc}
\hline \hline & Dim. & Rep. group & Exclusion principle & Origin of anyonic behavior \\
\hline Topological & 2D & Braid (Abelian) & Hard core for all $v \neq 0$ & Complex phase introduced by exchange \\
Statistical & Arbitrary & Permutation & Generalized exclusion & $\begin{array}{c}\text { Statistical average of bosonic and fermionic behavior } \\
\text { GES }\end{array}$ \\
Arbitrary & Permutation & Generalized exclusion & $\begin{array}{c}\text { intion of state occupancy arising from } \\
\text { interparticle interactions }\end{array}$ \\
\hline \hline
\end{tabular}


The Hamiltonian for our system of harmonically confined noninteracting statistical anyons reads

$$
H=\frac{p_{1}^{2}+p_{2}^{2}}{2 m}+\frac{1}{2} m \omega^{2}\left(x_{1}^{2}+x_{2}^{2}\right) .
$$

In the statistical anyon framework the partition function for a pair of anyons evolving under this Hamiltonian is

$$
Z_{\mathrm{SA}}=\left(Z_{B}\right)^{p_{B}}\left(Z_{F}\right)^{1-p_{B}},
$$

where $Z_{B}$ is the partition function for two bosons and $Z_{F}$ is the partition function for two fermions. See Appendix A for a full derivation of the partition function. Note that, as expected, Eq. (26) is identical to that of a GES CalogeroSutherland model gas [14], with the generalized exclusion statistics parameter $g$ replaced by $1-p_{B}$. We see that, in the statistical anyon framework, the anyonic behavior arises purely out of the average properties, rather than from an interaction term in the Hamiltonian.

Using Eq. (26) we can determine the internal energy, free energy, entropy, and heat capacity as follows:

$$
\begin{aligned}
& E=-\frac{\partial}{\partial \beta} \ln \left(Z_{\mathrm{SA}}\right), \quad F=-\frac{1}{\beta} \ln \left(Z_{\mathrm{SA}}\right), \\
& S=k_{B} \beta^{2} \frac{\partial F}{\partial \beta}, \quad C=-k_{B} \beta^{2} \frac{\partial E}{\partial \beta},
\end{aligned}
$$

where $\beta$ is the inverse temperature and $k_{B}$ is Boltzmann's constant. Plugging Eq. (26) into Eq. (27) we find

$$
\begin{aligned}
E= & \frac{1}{2} \hbar \omega\left[3 \operatorname{coth}(\beta \hbar \omega)+\operatorname{csch}(\beta \hbar \omega)-2 p_{B}+1\right], \\
F= & \frac{1}{\beta} \ln \left[\frac{1}{8} \operatorname{csch}^{2}\left(\frac{\beta \hbar \omega}{2}\right)-\frac{1}{4} \operatorname{csch}(\beta \hbar \omega)\right]-p_{B} \hbar \omega, \\
S= & \frac{1}{2} k_{B} \beta \hbar \omega[3 \operatorname{coth}(\beta \hbar \omega)+\operatorname{csch}(\beta \hbar \omega)+1], \\
& +k_{B} \ln \left[\frac{1}{8} \operatorname{csch}^{2}\left(\frac{\beta \hbar \omega}{2}\right)-\frac{1}{4} \operatorname{csch}(\beta \hbar \omega)\right], \\
C= & \frac{1}{2} k_{B} \beta^{2} \hbar^{2} \omega^{2} \operatorname{csch}^{2}(\beta \hbar \omega)[\cosh (\beta \hbar \omega)+3] .
\end{aligned}
$$

Plots of each as a function of temperature are shown in Fig. 3. We see that both the internal energy and free energy
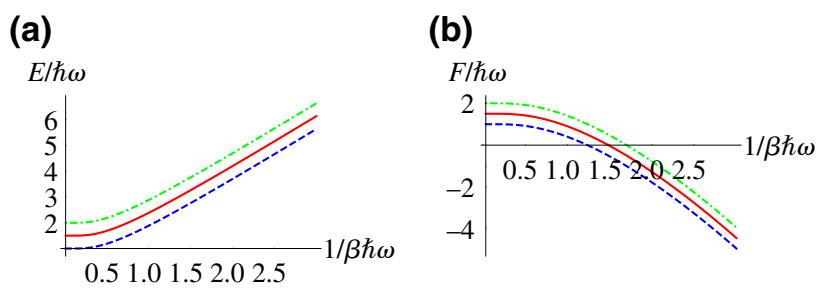

are shifted by a constant proportional to $p_{B}$. Physically this offset arises from the generalized exclusion principle, as outlined in Sec. II. The constant shifts the lowest energy state of the system from the bosonic limit, with both particles in the ground state of the oscillator, to the fermionic limit, with one particle in the ground state and the other in the first excited state.

We find that the dependence on $p_{B}$ cancels out exactly in the entropy and heat capacity, leaving them independent of the anyonic phase. This is expected, as in the thermal equilibrium state both fermions and bosons have an equivalent, countably infinite, number of available states. Note that the behavior of the heat capacity is consistent with that of a one-dimensional, two-oscillator Einstein solid [96]. However, since topological anyons do not exist in one dimension, in order to properly compare their thermodynamic behavior to that of statistical anyons, we must extend the above analysis to two dimensions.

\section{B. 2D statistical anyons}

We can repeat the thermodynamic analysis for two statistical anyons in a two-dimensional harmonic potential. To avoid clutter, we give the full expressions for the internal energy, free energy, entropy, and heat capacity in Appendix B. We plot each as a function of temperature in Fig. 4. In contrast to the one-dimensional case, we see that now the entropy and heat capacity do depend on $p_{B}$. The origin of this difference is clear if we think of the entropy for bosons and fermions in the zero-temperature limit. For bosons, only one configuration is available - both particles in the ground state in both dimensions. However, the fermion ground state is degenerate. The Pauli exclusion principle requires that one particle must be in the ground state and the other in the first excited state, but the excited state can be in either dimension. This results in a nonzero entropy at $T=0$. The entropy of generic statistical anyons interpolates smoothly between the boson and fermion limits as $p_{B}$ changes from 1 to 0 .

\section{2D topological anyons}

The partition function for two topological anyons in a two-dimensional harmonic potential has been previously

(c)

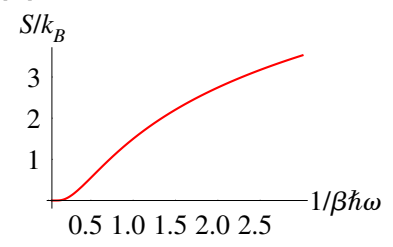

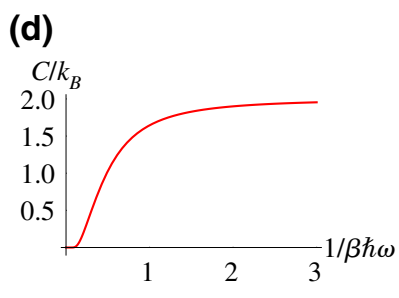

FIG. 3. Equilibrium (a) internal energy, (b) free energy, (c) entropy, and (d) heat capacity for two statistical anyons in one dimension with anyonic phase corresponding to $p_{B}=1$ (blue, dashed), $p_{B}=1 / 2$ (red, solid), and $p_{B}=0$ (green, dot dashed). 
(a)

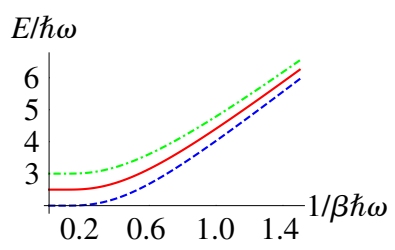

(b)

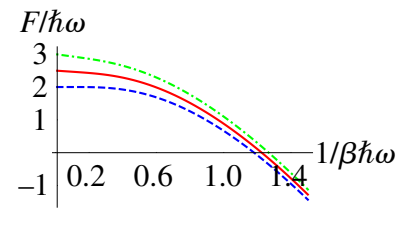

(c)

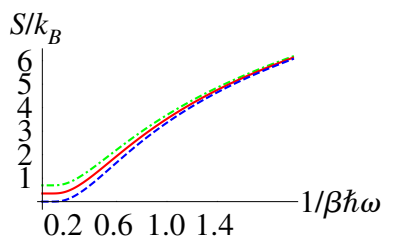

(d)

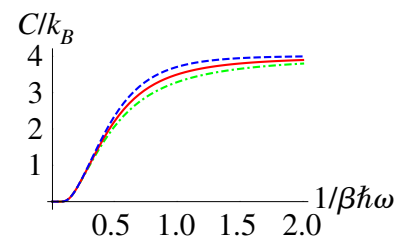

FIG. 4. Equilibrium (a) internal energy, (b) free energy, (c) entropy, and (d) heat capacity for two statistical anyons in two dimensions with anyonic phase corresponding to $p_{B}=1$ (blue, dashed), $p_{B}=1 / 2$ (red, solid), and $p_{B}=0$ (green, dot dashed).

derived (see, for example, Refs. [51,56]). It is given by

$$
Z_{\mathrm{TA}}=\frac{e^{-\beta \hbar \omega(2+v)}+e^{-\beta \hbar \omega(4-v)}}{\left(1-e^{-\beta \hbar \omega}\right)^{2}\left(e^{-2 \beta \hbar \omega}\right)^{2}},
$$

where $v$ is the anyonic phase. The first thing we note is that, unlike the statistical anyon and GES anyon partition function, the topological anyon partition function cannot be expressed as a function of the boson and fermion partition functions. This arises from the increased complexity of the topological anyon wave function. In the two-anyon problem, the anyonic phase can be considered as a shift in the value of the relative motion angular momentum [43]. However, accounting for the fact that the phase depends on the direction of rotation (a consideration unique to the braid group-based topological anyons) leads to a multivalued wave function [51]. Accounting for both branches of the wave function results in the two separate anyonic phase-dependent terms seen in the numerator of Eq. (29).

Following the same process as for the statistical anyons, we determine the equilibrium internal energy, free energy, entropy, and heat capacity for the two-anyon system, with the full expressions given in Appendix B. Plots of each as a function of temperature are given in Fig. 5. Here we see qualitatively similar behavior to the two-dimensional statistical anyons, with some notable differences. We see that the entropy of topological anyons converges to zero in the zero-temperature limit, indicating the existence of a unique ground-state configuration for all values of $v$ except $v=1$, corresponding to pure fermions. Another significant discrepancy is seen in the behavior of the heat capacity.
For intermediate values of the anyonic phase we see that, at low temperatures, the topological anyon heat capacity is higher than that of both the bosonic and fermionic values.

Comparing Figs. 4 and 5 we see that, in each plot, for the statistical anyons the line corresponding to $p_{B}=1 / 2$ remains evenly spaced between the bosonic and fermionic limits, while for topological anyons the line corresponding to $v=1 / 2$ bends more towards the fermionic behavior. This difference has its origin in the complicated energy spectrum that arises from the topological anyons' "hardcore" exclusion principle and multivalued wave function. For topological anyons in a harmonic potential, the energy, degeneracy, and level spacing all depend on $v$ [43,51]. In the ground state, the energy eigenvalues corresponding to the two branches of the wave function coincide only in the fermionic limit, making $v=1$ the only degenerate ground state and giving rise to the observed zero-temperature limit of the entropy. However, as temperature increases, the hard-core nature of topological anyons biases their thermodynamic behavior more towards the fermionic limit.

The unique behavior of the topological anyon heat capacity arises from similar considerations. In the zerotemperature limit, the internal energy of the topological anyons interpolates linearly between the bosonic and fermionic ground states for intermediate values of $v$, while at higher temperatures it converges to the fermionic behavior. This transition between an even average of the bosonic and fermionic behavior at zero temperature to near-fermionic behavior at higher temperatures necessitates a steeper positive slope of the internal energy at low temperatures than either bosons or fermions. This, in turn, (a)

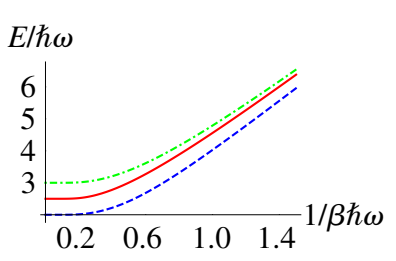

(b)

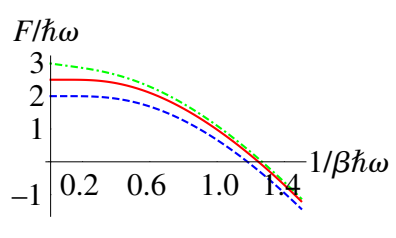

(c)

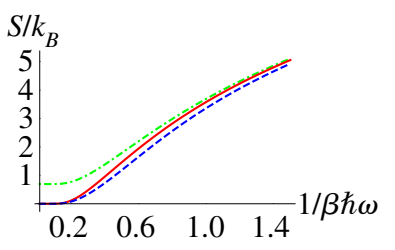

(d)

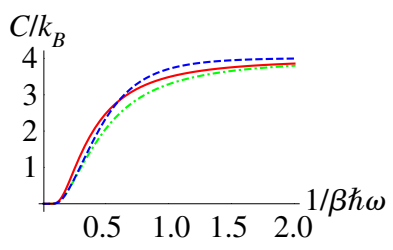

FIG. 5. Equilibrium (a) internal energy, (b) free energy, (c) entropy, and (d) heat capacity for two topological anyons with anyonic phase corresponding to $v=0$ (blue, dashed), $v=1 / 2$ (red, solid), and $v=1$ (green, dot dashed). 
leads to a heat capacity for intermediate values of $v$ that exceeds both bosons and fermions in this low-temperature transition region.

In contrast, the statistical anyon energy spectrum is a much simpler weighted average over the bosonic and fermionic spectrums, leading to thermodynamic behavior that is evenly spaced between the bosonic and fermionic limits over all temperatures for $p_{B}=1 / 2$. This has notable ramifications for thermodynamic applications of anyons, as statistical anyons will retain their "intermediate" behavior at higher temperature regimes.

\section{Thermodynamic equivalence}

In this section we have seen that statistical and topological anyons display different thermodynamic behavior. This brings up the question: Is it possible to mimic the richer behavior of topological anyons using the mathematically and experimentally simpler framework of statistical anyons?

To determine the relation between the topological anyon parameter $v$, and the statistical anyon parameter $p_{B}$ we can set their partition functions equal to each other and solve for $p_{B}$. This yields

$$
p_{B}=\frac{\ln \{\cosh [(\nu-1) \beta \hbar \omega]\}}{\ln [\cosh (\beta \hbar \omega)]} .
$$

We see that in order to capture the more complicated thermodynamic behavior $p_{B}$ becomes dependent on the temperature and frequency parameters. If we take the high-temperature limit of Eq. (30) we find a simpler, parameter-independent relation,

$$
p_{B}=(v-1)^{2} .
$$

We note that the approximate relation becomes exact for $v=0,1$, as the behavior of the statistical and topological anyons must converge to the same bosonic and fermionic limits. Notably, previous comparisons between Haldane's GES parameter and the topological anyon phase using the second Virial coefficient have also determined a quadratic polynomial relation [12].

Plotting the internal energy, free energy, entropy and heat capacity for topological anyons and statistical anyons using Eq. (31) in Fig. 6 we see that the statistical and topological anyon behavior rapidly converges. We note that the parameter-independent approximation does not capture the low-temperature behavior of the topological anyon heat capacity. To fully imitate this behavior, we must use Eq. (30).

By applying Eq. (27) along with the relation in Eq. (30) it is straightforward to see that the equations of state for statistical and topological anyons become identical. The ability to mimic the thermodynamic properties of topological anyons using statistical anyons has important ramifications from an experimental standpoint. The difficulty of detecting and manipulating topological anyons in two-dimensional materials makes probing their thermodynamics exceedingly challenging. Statistical anyons provide a straightforward alternative, both as a model to test thermodynamic control of topological anyons or as a replacement in applications that would rely on their thermodynamics properties.

\section{ENDOREVERSIBLE ANYONIC ENGINE}

Having established the equilibrium thermodynamic behavior of both statistical and topological anyons, we now continue our exploration of their behavior in the context of heat engines. Studying the thermodynamic properties of a system using the framework of cyclic heat engines has a rich tradition as old as thermodynamics itself [60]. In equilibrium thermodynamics the optimal efficiency of any heat-engine cycle is bounded by the Carnot efficiency, regardless of the properties of the working medium [97]. However, this efficiency is obtained in the limit of infinitely slow, quasistatic strokes, resulting in zero power output. A figure of merit of more practical use, the efficiency at maximum power (EMP), was introduced by Curzon and Ahlborn using the framework of endoreversible thermodynamics [98-100]. Curzon and Ahlborn found the EMP of a endoreversible Carnot engine to be

$$
\eta_{\mathrm{CA}}=1-\sqrt{\frac{T_{c}}{T_{h}}},
$$

where $T_{c}\left(T_{h}\right)$ is the cold (hot) reservoir temperature [98].
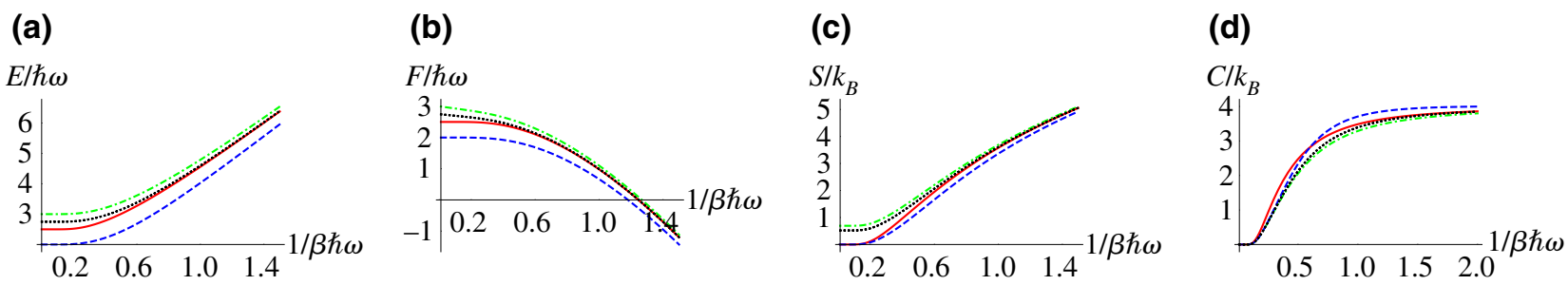

FIG. 6. Comparison of (a) internal energy, (b) free energy, (c) entropy, and (d) heat capacity of two topological anyons with phase parameter $v$, against two statistical anyons using phase parameter $p_{B}=(v-1)^{2}$. The lines correspond to $v=0, p_{B}=1$ (blue, dashed), $v=1, p_{B}=0$ (green, dot dashed), $v=1 / 2$ (red, solid), and $p_{B}=1 / 4$ (black, dotted). 
In endoreversible thermodynamics the system is assumed to be in a state of local equilibrium at all times, but with dynamics that occur quickly enough that global equilibrium with the environment is not achieved. This results in a process that is locally reversible, but globally irreversible [100]. It has been shown that the performance of a quantum Otto engine is dependent on the stroke protocol [101-114] and the nature of the working medium [115-129], with the EMP, in particular, being determined by the form of the fundamental relation of the working medium [130]. In Ref. [122] it was shown that the EMP of an endoreversible quantum Otto engine with a singleparticle working medium can exceed the Curzon-Ahlborn efficiency. It is of interest then to examine the role of quantum statistics in the operation of such an engine.

In the following analysis we closely follow the method established in Ref. [122]. Let us consider a working medium of two anyons in a harmonic potential, evolving under the Hamiltonian given in Eq. (25) with a timedependent frequency. The Otto cycle consists of four strokes summarized graphically in Fig. 7.

(1) Isentropic compression

During this stroke the working medium remains in a state of constant entropy, exchanging no heat with the environment. Using the first law $\Delta E=Q+W$ we can identify the change in internal energy completely with work,

$$
W_{\text {comp }}=E\left(T_{B}, \omega_{2}\right)-E\left(T_{A}, \omega_{1}\right) .
$$

\section{(2) Isochoric heating}

During this stroke the externally controlled work parameter (the trap frequency in the case of the harmonic engine) is held constant, resulting in zero work. By the first law we

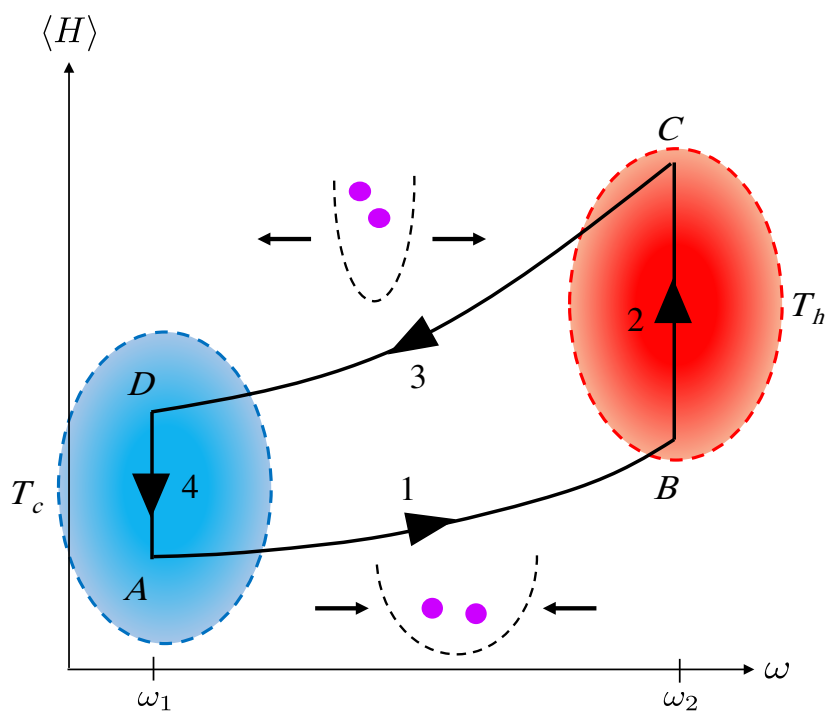

FIG. 7. Energy-frequency diagram of a quantum Otto cycle for a harmonic trapping potential with a working medium of two anyons. can then identify the change in internal energy completely with heat,

$$
Q_{h}=E\left(T_{C}, \omega_{2}\right)-E\left(T_{B}, \omega_{2}\right)
$$

Recalling the conditions of endoreversibility we note that the working medium does not fully thermalize with the hot reservoir during this stroke, giving us the condition $T_{B} \leq$ $T_{C} \leq T_{h}$ [98]. The change in temperature during the stroke depends on the properties of the working medium can be determined using Fourier's law [97],

$$
\frac{d T}{d t}=-\alpha_{h}\left[T(t)-T_{h}\right]
$$

where $\alpha_{h}$ is a constant determined by the heat capacity and thermal conductivity of the working medium.

(3) Isentropic expansion

In exactly the same manner as the compression stroke, we can identify the change in internal energy during the expansion with work,

$$
W_{\exp }=E\left(T_{D}, \omega_{1}\right)-E\left(T_{C}, \omega_{2}\right)
$$

\section{(4) Isochoric cooling}

As in the heating stroke, we identify the change in internal energy during this stroke with heat,

$$
Q_{c}=E\left(T_{A}, \omega_{1}\right)-E\left(T_{D}, \omega_{1}\right)
$$

The temperature change can again be determined from Fourier's law,

$$
\frac{d T}{d t}=-\alpha_{c}\left[T(t)-T_{c}\right]
$$

where $T_{D}>T_{A} \geq T_{c}$.

The efficiency of the engine is given by the ratio of the total work and the heat exchanged with the hot reservoir,

$$
\eta=-\frac{W_{\mathrm{comp}}+W_{\mathrm{exp}}}{Q_{h}},
$$

and the power output by the ratio of the total work to the cycle duration,

$$
P=-\frac{W_{\mathrm{comp}}+W_{\mathrm{exp}}}{\gamma\left(\tau_{h}+\tau_{c}\right)} .
$$

Note that only the durations of the heating and cooling strokes are accounted for explicitly, with $\gamma$ serving as a multiplicative factor that implicitly incorporates the duration of the isentropic strokes [122]. 


\section{A. 1D statistical anyons}

Combining the internal energy and entropy from Eq. (28) with Eqs. (33), (34), and (36) and plugging it all into Eq. (39) yields a complicated expression that can be considerably simplified. First we note that from the isentropic strokes we have the conditions

$$
S\left(T_{A}, \omega_{1}\right)=S\left(T_{B}, \omega_{2}\right) \text { and } S\left(T_{C}, \omega_{2}\right)=S\left(T_{D}, \omega_{1}\right) .
$$

Using Eq. (28) it is straightforward to verify that the conditions in Eq. (41) are satisfied by

$$
T_{A} \omega_{2}=T_{B} \omega_{1} \text { and } T_{C} \omega_{1}=T_{D} \omega_{2} .
$$

Furthermore, Eqs. (35) and (38) can be fully solved to yield,

$$
\begin{aligned}
T_{C}-T_{h} & =\left(T_{B}-T_{h}\right) e^{-\alpha_{h} \tau_{h}}, \\
T_{A}-T_{c} & =\left(T_{D}-T_{c}\right) e^{-\alpha_{c} \tau_{c}},
\end{aligned}
$$

where $\tau_{h}\left(\tau_{c}\right)$ is the duration of the heating (cooling) stroke. Combining Eq. (39) with Eqs. (42) and (43) yields a much simplified form for the efficiency,

$$
\eta=1-\kappa,
$$

where $\kappa \equiv \omega_{1} / \omega_{2}$ is the compression ratio. We note that this efficiency is identical to classical, reversible Otto efficiency, as well as the single-particle quantum Otto efficiency found in Ref. [122] and is completely independent of the quantum statistics of the working medium.

To find the EMP we next need to compute the power, given by Eq. (45). Eliminating free parameters using the same simplification process as we did for the efficiency we arrive at the much more cumbersome expression,

$$
\begin{aligned}
P= & \frac{(1-\kappa) \omega_{2} \hbar}{\gamma\left(\tau_{h}+\tau_{c}\right)}[3 \operatorname{coth}(\Gamma)-3 \operatorname{coth}(\Lambda) \\
& +\operatorname{csch}(\Gamma)-\operatorname{csch}(\Lambda)]
\end{aligned}
$$

where

$$
\begin{aligned}
\Gamma & =\frac{\kappa \omega_{2} \hbar\left(e^{\alpha_{c} \tau_{c}+\alpha_{h} \tau_{h}}-1\right)}{k_{B}\left[\kappa T_{h}\left(e^{\alpha_{h} \tau_{h}}-1\right) e^{\alpha_{c} \tau_{c}}+T_{c}\left(e^{\alpha_{c} \tau_{c}}-1\right)\right]}, \\
\Lambda & =\frac{\kappa \omega_{2} \hbar\left(e^{\alpha_{c} \tau_{c}+\alpha_{h} \tau_{h}}-1\right)}{k_{B}\left[\kappa T_{h}\left(e^{\alpha_{h} \tau_{h}}-1\right)+T_{c}\left(e^{\alpha_{c} \tau_{c}}-1\right) e^{\alpha_{h} \tau_{h}}\right]} .
\end{aligned}
$$

The first thing that we can note about this expression is that the endoreversible power output does not depend on the statistics of the working medium for one-dimensional statistical anyons. This is consistent with the results of Ref. [127], which showed differences in the performance of a one-dimensional harmonic quantum Otto engine arising from the bosonic or fermionic nature of the working medium are a feature of nonequilibrium performance. In the case of endoreversible operation, the only effect of the generalized exclusion principle is to shift the value of the work expended during the compression stroke by $\hbar p_{B}\left(\omega_{1}-\omega_{2}\right)$ and the work extracted during the expansion stroke by $\hbar p_{B}\left(\omega_{2}-\omega_{1}\right)$. Thus when $W_{\text {comp }}$ and $W_{\exp }$ are summed to determine the total work, these contributions exactly cancel each other out.

We observe that the power vanishes for the case of $\Gamma=\Lambda$. Examining Eq. (46) we see that these expressions become equivalent in the limit $\kappa \rightarrow T_{c} / T_{h}$. This matches with our physical intuition, as this limit corresponds to quasistatic operation at Carnot efficiency. This provides us with the parameter range $\Gamma<\Lambda$ where the power output is positive and the cycle operates as an engine. This is typically referred to as the positive work condition.

To find the EMP we maximize Eq. (45) numerically with respect to the compression ratio. The EMP as a function of the ratio of bath temperatures for one-dimensional statistical anyons is shown in Fig. 8, along with the EMP of distinguishable particles, the Curzon-Ahlborn efficiency, and the Carnot efficiency for comparison. Notably we see that, while the EMP does not depend on the anyonic phase, it does depend on whether or not the particles of the working medium are distinguishable. In this case, distinguishable refers to particles that remain sufficiently spatially separated such that the overlap of their wave functions is negligible, negating any behavior that would arise from the exchange forces. Experimentally, we can consider an engine consisting of two distinguishable particles as equivalent to the joint output of two separate singleparticle engines situated across the lab from each other.

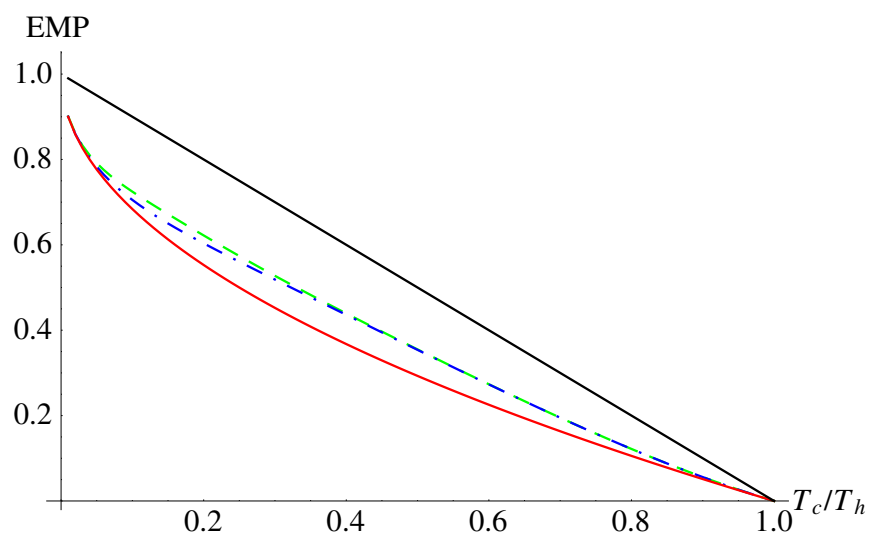

FIG. 8. EMP as a function of the ratio of bath temperatures for two distinguishable quantum particles (dot dashed, blue) and two indistinguishable statistical anyons (dashed, green) in one dimension. The Curzon-Ahlborn efficiency (bottom solid, red) and the Carnot efficiency (top solid, black) are given in comparison. Operation is in the quantum regime corresponding to $\hbar \omega_{2} / k_{B} T_{c}=10$. Parameters are $\alpha_{c}=\alpha_{h}=\gamma=1$, and $\tau_{c}=\tau_{h}=0.5$. 
We see that, at low temperature ratios, the EMP of the indistinguishable, anyonic working medium outperforms that of two distinguishable quantum particles. We also note that for both indistinguishable and distinguishable quantum working mediums the EMP is greater than the Curzon-Ahlborn efficiency, confirming the results found in Ref. [122].

\section{B. 2D statistical anyons}

It is straightforward to extend the previous analysis to two dimensions. As noted in Sec. IV, in two dimensions the entropy is no longer independent of the anyonic phase, in the form of $p_{B}$. Similarly, in the two-dimensional endoreversible engine the $p_{B}$ dependence in the work done on expansion and expended during compression no longer cancel each other out, resulting in an anyonic phase-dependent power output,

$$
\begin{aligned}
P= & \frac{(1-\kappa) \omega_{2} \hbar}{\gamma\left(\tau_{h}+\tau_{c}\right)}[2 \operatorname{coth}(\Gamma)-2 \operatorname{coth}(\Lambda)+\operatorname{coth}(\Gamma / 2) \\
& \left.-\operatorname{coth}(\Lambda / 2)+p_{B} \tanh (\Lambda)-p_{B} \tanh (\Gamma)\right],
\end{aligned}
$$

where $\Lambda$ and $\Gamma$ are the same as given in Eq. (46). This dependence manifests in the EMP at small values of the bath-temperature ratio, shown in Fig. 9. We see that, as in one dimension, all indistinguishable working mediums show greater EMP than a working medium of two distinguishable quantum particles. We see further that the

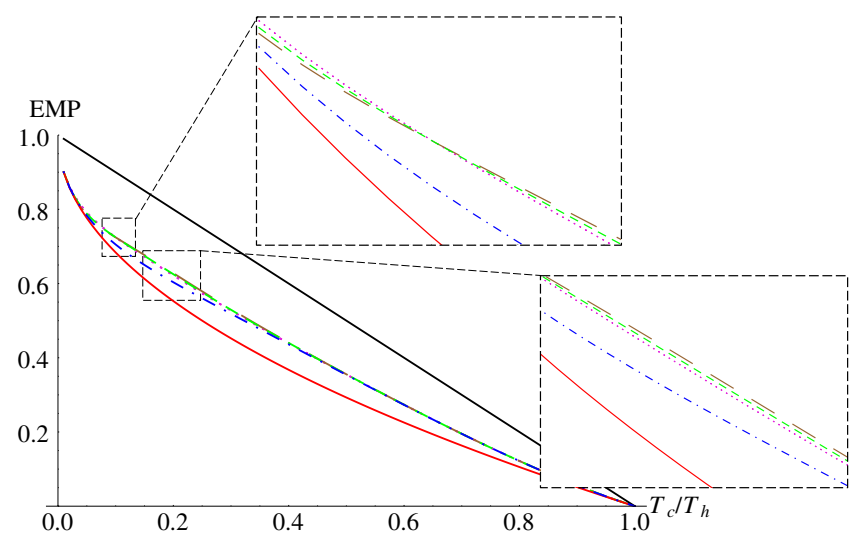

FIG. 9. EMP as a function of the ratio of bath temperatures for two statistical anyons with $p_{B}=1$ (long dashed, brown), $p_{B}=1 / 2$ (short dashed, green), $p_{B}=0$ (dotted, dark pink), and two distinguishable quantum particles (dot dashed, blue) in two dimensions. The Curzon-Ahlborn efficiency (solid, red) is given in comparison. The bottom inset highlights the range of bathtemperature ratios at which bosonic working mediums display the greatest EMP and the top inset highlights the critical point and transition to the region where fermionic working mediums begin to outperform bosonic ones. Operation is in the quantum regime corresponding to $\hbar \omega_{2} / k_{B} T_{c}=10$. Parameters are $\alpha_{c}=\alpha_{h}=\gamma=1$, and $\tau_{c}=\tau_{h}=0.5$. anyonic phase that gives maximum performance depends on the bath temperature ratio. For temperature ratios between around 0.1 and 0.25 we see that bosonic symmetry $\left(p_{B}=1\right)$ gives the greatest enhancement to EMP over distinguishable particles and fermionic symmetry $\left(p_{B}=0\right)$ the least. However, around $T_{c} / T_{h}=0.1$ there is a crossing, after which the fermionic symmetry gives the greatest enhancement to the EMP and bosonic symmetry the least. Intermediate values of $p_{B}$ interpolate smoothly between these limits. Interestingly, this transition indicates the existence of a critical point at which the EMP becomes equivalent for all values of $p_{B}$.

The origin of this behavior can be traced to the fact that, unlike in the one-dimensional case, the energy shift arising from the generalized exclusion principle is temperature dependent. In one dimension, while the magnitude of each energy eigenvalue depends on whether the particles are bosons or fermions, the degeneracy does not. In two dimensions, both the magnitude and degeneracy of each energy state differ between bosons and fermions, leading to the temperature-dependent shift in the internal energy. Due to this temperature dependence, in general the contributions to the work from $p_{B}$ on the compression and expansion strokes no longer cancel out. The critical point then corresponds to the unique ratio of bath temperatures such that these contributions become exactly equal, leading to $p_{B}$-independent performance.

\section{2D topological anyons}

Using the partition function in Eq. (29) we can carry out the endoreversible analysis for a harmonic quantum Otto engine with a working medium of topological anyons. This results in an expression for the power that is very similar to the two-dimensional statistical anyon power, but with an additional factor dependent on the anyonic phase within the phase-dependent hyperbolic trigonometric terms,

$$
\begin{aligned}
P= & \frac{(1-\kappa) \omega_{2} \hbar}{\gamma\left(\tau_{h}+\tau_{c}\right)}\{2 \operatorname{coth}(\Gamma)-2 \operatorname{coth}(\Lambda)+\operatorname{coth}(\Gamma / 2) \\
& -\operatorname{coth}(\Lambda / 2)+(1-v) \tanh [(1-v) \Lambda] \\
& -(1-v) \tanh [(1-v) \Gamma]\} .
\end{aligned}
$$

This results in richer behavior, with the power no longer always being maximized by either the bosonic or fermionic limit. Figure 10 shows the power as a function of the anyonic phase for several different bath-temperature ratios. We see that as the bath-temperature ratio increases, the anyonic phase that maximizes the power output shifts from more fermionic to more bosonic.

Again maximizing with respect to the frequency, we find an EMP with complex dependence on the anyonic phase, shown in Fig. 11. Consistent with the pure power, we see that the anyonic EMP is no longer bounded by the bosonic 


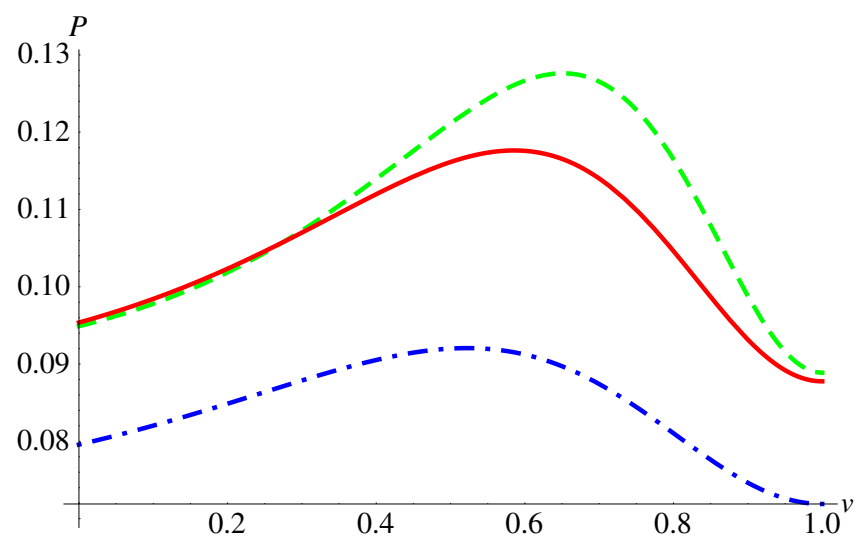

FIG. 10. Power as a function of the anyonic phase for two topological anyons at $T_{c} / T_{h}=0.1$ (dashed, green), $T_{c} / T_{h}=$ 0.2 (solid, red), and $T_{c} / T_{h}=0.3$ (dot dashed, blue). Parameters are $\alpha_{c}=\alpha_{h}=\gamma=\tau_{c}=\tau_{h}=1$, and $\kappa=0.5$, along with $\hbar=k_{B}=1$.

and fermionic limits of the anyonic phase. The phase that provides the maximum EMP is highly temperature dependent, and we see intermediate values that provide both better and worse EMP than either bosons or fermions. Furthermore, the anyonic EMP can even fall below that of the distinguishable particles. One such example for the case of $v=0.8$ is shown in Fig. 12 .

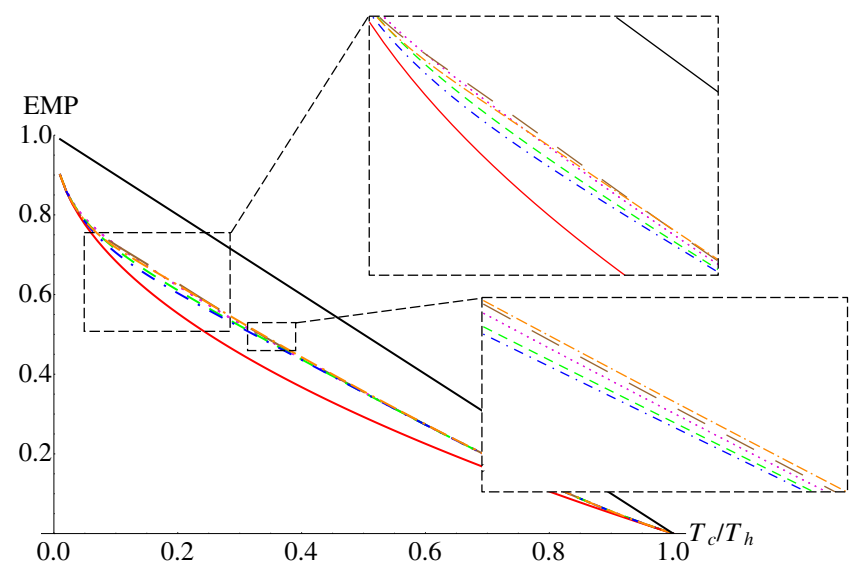

FIG. 11. EMP as a function of the ratio of bath temperatures for two topological anyons with $v=0$ (long dashed, brown), $v=$ $1 / 2$ (short dashed, green), $v=0.25$ (dot dash dashed, orange), $v=1$ (dotted, dark pink), and two distinguishable quantum particles (dot dashed, blue) in two dimensions. The CurzonAhlborn efficiency (bottom solid, red) and Carnot efficiency (top solid, black) are given in comparison. The bottom inset highlights a parameter region where an intermediate anyonic phase $(v=0.25)$ displays the greatest EMP and the top inset highlights a region of low-temperature ratios that exhibits multiple crossings. Operation is in the quantum regime corresponding to $\hbar \omega_{2} / k_{B} T_{c}=10$. Parameters are $\alpha_{c}=\alpha_{h}=\gamma=1$, and $\tau_{c}=$ $\tau_{h}=0.5$.

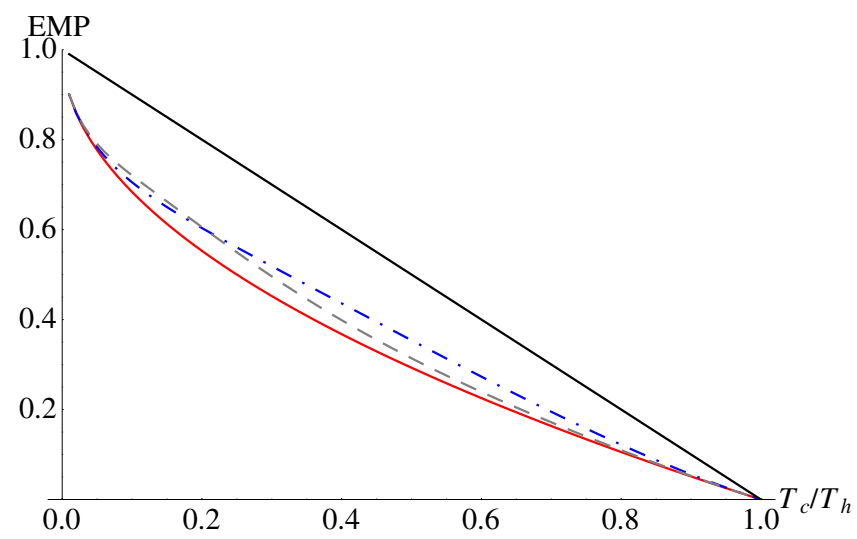

FIG. 12. EMP as a function of the ratio of bath temperatures for two topological anyons with $v=0.8$ (dashed, gray) and two distinguishable quantum particles (dot dashed, blue). The Curzon-Ahlborn efficiency (lower solid, red) and Carnot efficiency (upper solid, black) are given in comparison. Operation is in the quantum regime corresponding to $\hbar \omega_{2} / k_{B} T_{c}=10$. Parameters are $\alpha_{c}=\alpha_{h}=\gamma=1$, and $\tau_{c}=\tau_{h}=0.5$.

The origins of this more complicated behavior become clear when comparing Eq. (47) and Eq. (48). We see that, as in the case of the two-dimensional statistical anyons, the energy shifts arising from the statistics no longer cancel out (except in the quasistatic limit of $\Gamma=\Lambda$ ), giving rise to the two $v$-dependent hyperbolic tangent terms in Eq. (48). However, the more complicated energy spectrum arising from the hard-core restriction and multivalued wave function results in an additional $v$ dependence in the argument of the trigonometric functions. This highly nonlinear dependence is responsible for the large variations in performance for intermediate values of $v$ seen in Figs. 11 and 12.

From the standpoint of pure performance, the existence of temperature regimes where intermediate values of $v$ exceed the bosonic EMP demonstrates an advantage for topological anyons over statistical anyons as a working medium. However, the complex dependence of the power on $v$ also has the consequence that small variations in the anyonic phase can lead to vastly different performance, as seen for the case of $v=0.8$. From a quantum metrology standpoint, the fact that performance is linked directly to the anyonic phase for both statistical and topological anyons indicates that thermal machines may be a useful tool for detecting signatures of anyonic statistics. We note that, using the parameter-dependent anyonic parameter in Eq. (30), it is possible to fully capture the behavior of a topological anyon heat engine using statistical anyons. As Eq. (30) yields the same equations of state for statistical and topological anyons, the endoreversible analysis for both mediums will be identical, leading to exactly equivalent performance. 


\section{ANYONIC ENGINE: BEYOND ENDOREVERSIBILITY}

Under the conditions of endoreversiblity we have seen that the engine performance depends on the anyonic phase for both statistical and topological anyons in two dimensions, but not for statistical anyons in one dimension. We now move beyond the assumption of local equilibrium, and consider a finite-time quantum Otto cycle with fully nonequilibrium isentropic strokes. To focus on the effects on engine performance arising solely from the quantum statistics, we make the standard assumption that the thermalization time is short enough and the isochoric strokes long enough that the working medium is in a state of thermal equilibrium with the hot (cold) bath at point $C$ $(A)$ in the cycle, removing the need to explicitly model the interaction with the heat baths $[102,104,105,107,127$, 128,131,132].

For the full nonequilibrium treatment we restrict our analysis to the case of one-dimensional statistical anyons, as of the three working mediums we have explored so far this was the only one that has not yet shown effects arising from the nature of the quantum statistics. In order to calculate the efficiency, power, and EMP of the engine, we must determine the internal energies at points $A, B, C$, and $D$ in the cycle. These can be found from the density operator in the typical fashion,

$$
\langle H\rangle=\operatorname{tr}\{\rho H\} .
$$

For $N$-independent particle pairs, the total density operator is given by the product of the individual density operators for each pair,

$$
\rho_{N}=\prod_{j=1}^{N_{B}} \rho_{B}^{(j)} \prod_{k=N_{B}+1}^{N} \rho_{F}^{(k)}
$$

where $\rho_{B}$ and $\rho_{F}$ are the density operators of particle pairs with bosonic and fermionic symmetry, respectively. Combining Eqs. (49) and (50) we have

$$
\begin{aligned}
\left\langle H_{N}\right\rangle & =\sum_{j=1}^{N_{B}} \operatorname{tr}\left\{\rho_{B}^{(j)} H_{j}\right\}+\sum_{k=N_{B}+1}^{N} \operatorname{tr}\left\{\rho_{F}^{(k)} H_{k}\right\} \\
& =N_{B}\left\langle H_{B}\right\rangle+\left(N-N_{B}\right)\left\langle H_{F}\right\rangle .
\end{aligned}
$$

Using $N_{B}=N p_{B}$ we arrive at the expression for the internal energy of a single pair of statistical anyons,

$$
\left\langle H_{\mathrm{SA}}\right\rangle=p_{B}\left\langle H_{B}\right\rangle+\left(1-p_{B}\right)\left\langle H_{F}\right\rangle .
$$

In Ref. [127] both the thermal state and time-evolved density operators are derived for two bosons and fermions in a harmonic potential (for completeness, the full expressions are provided in Appendix C). The corresponding internal energies are

$$
\begin{aligned}
\langle H\rangle_{A} & =\frac{\hbar \omega_{1}}{2}\left[3 \operatorname{coth}\left(\beta_{1} \hbar \omega_{1}\right)+\operatorname{csch}\left(\beta_{1} \hbar \omega_{1}\right) \mp 1\right], \\
\langle H\rangle_{B} & =\frac{\hbar \omega_{2}}{2} Q_{12}^{*}\left[3 \operatorname{coth}\left(\beta_{1} \hbar \omega_{1}\right)+\operatorname{csch}\left(\beta_{1} \hbar \omega_{1}\right) \mp 1\right], \\
\langle H\rangle_{C} & =\frac{\hbar \omega_{2}}{2}\left[3 \operatorname{coth}\left(\beta_{2} \hbar \omega_{2}\right)+\operatorname{csch}\left(\beta_{2} \hbar \omega_{2}\right) \mp 1\right], \\
\langle H\rangle_{D} & =\frac{\hbar \omega_{1}}{2} Q_{21}^{*}\left[3 \operatorname{coth}\left(\beta_{2} \hbar \omega_{2}\right)+\operatorname{csch}\left(\beta_{2} \hbar \omega_{2}\right) \mp 1\right] .
\end{aligned}
$$

Here the $(-)$ corresponds to bosons and the $(+)$ to fermions. $Q_{12}^{*}$ and $Q_{21}^{*}$ are protocol-dependent dimensionless parameters that measure the degree of adiabaticity of the isentropic strokes [133]. Using Eqs. (52) and (53) we can determine the engine efficiency and power output. The full expressions are cumbersome and given in Appendix D. We note that the engine behavior we find here is equivalent to that found in Ref. [119] for an Otto engine with a working medium of a Calogero-Sutherland gas. However, our underlying construction is very different, with the anyonic nature of the working medium arising from a simple statistical average over bosons and fermions rather than an additional interparticle interaction term.

To continue our analysis we must pick a specific protocol for the compression and expansion strokes. For simplicity we choose the "sudden switch" protocol, which corresponds to an instantaneous quench from the initial to final frequency. For the sudden switch the adiabaticity parameters are

$$
Q_{12}^{*}=Q_{21}^{*}=\frac{1+\kappa^{2}}{2 \kappa},
$$

where $\kappa=\omega_{1} / \omega_{2}$.

Following the same method of performance analysis that we used in the endoreversible case, we numerically maximize the power with respect to the frequency ratio in order to determine the EMP. The EMP as a function of the bathtemperature ratios is shown in Fig. 13. We immediately see that in the case of nonequilibrium operation the performance is no longer independent of the anyonic phase. We note fermionic symmetry gives the worst EMP and bosonic symmetry the best, with intermediate values of $p_{B}$ falling between. In the parameter regimes explored we see no transitions between anyonic phases that provides the optimal EMP, unlike the two-dimensional endoreversible engines.

In general we see that the EMP of the nonequilibrium engine is significantly worse than in the endoreversible case, no longer outperforming the Curzon-Ahlborn efficiency. This is unsurprising, as the sudden switch protocol we have employed is far from adiabatic, resulting 
(a)

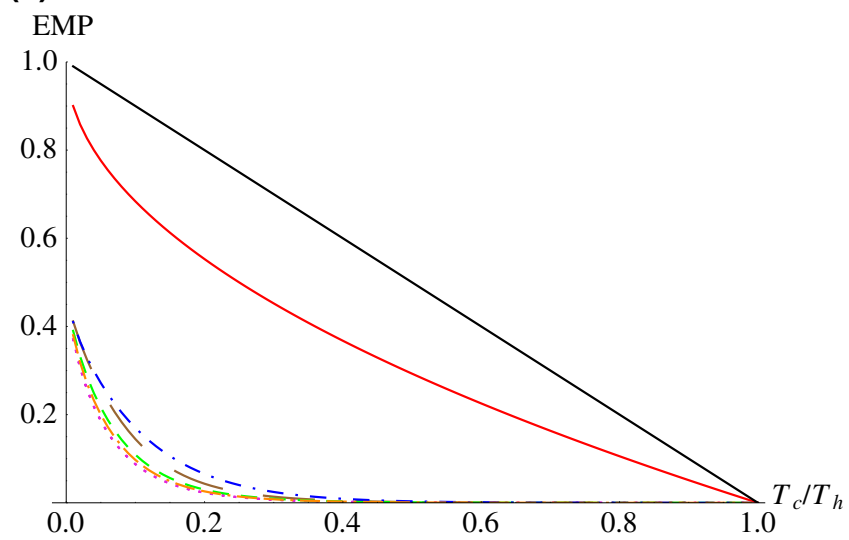

(b)

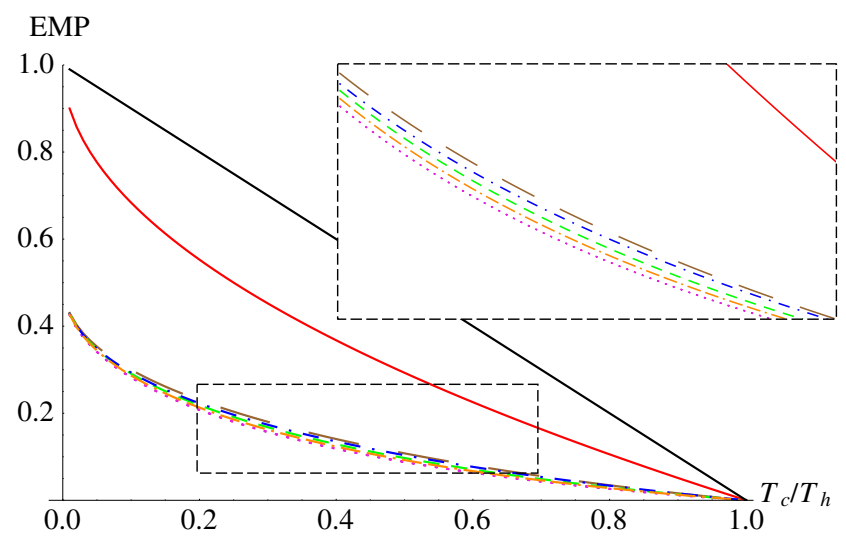

FIG. 13. Nonequilibrium EMP as a function of the ratio of bath temperatures for two statistical anyons in one dimension with $p_{B}=1$ (long dashed, brown), $p_{B}=1 / 2$ (short dashed, green), $p_{B}=0.25$ (dot dash dashed, orange), $p_{B}=0$ (dotted, dark pink), and two distinguishable quantum particles (dot dashed, blue). The Curzon-Ahlborn efficiency (lower solid, red) and Carnot efficiency (top solid, black) are given in comparison. Plot (a) shows operation in the regime corresponding to $\hbar \omega_{2} / k_{B} T_{c}=10$ and (b) in the regime corresponding to $\hbar \omega_{2} / k_{B} T_{c}=1$. We set $\tau=1$.

in significantly lower power due to the loss of energy to nonadiabatic excitations. We also compare the EMP as we transition from the deep quantum regime characterized by $\hbar \omega_{2} / k_{B} T_{c}=10$ to a more classical regime characterized by $\hbar \omega_{2} / k_{B} T_{c}=1$. We see that in the deep quantum regime the indistinguishable particles give worse performance than distinguishable quantum particles. As we transition toward the more classical regime the gaps in EMP between different values of the anyonic phase shrink, but we also see that the indistinguishable particles begin to outperform the distinguishable ones. Figure 13(b) examines the engine performance in the same parameter regime studied in Ref. [127], where we see the same bosonic advantage emerge.

In the case of topological anyons it seems likely that, as in the statistical anyon case, moving to the fully nonequilibrium regime would lead to significantly lower EMP due to nonadiabatic excitations. However, verifying this would require solving the full time-dependent Schrödinger equation for two topological anyons in a two-dimensional parametric harmonic potential. This is a challenging problem that we hope may be explored in future work, but is beyond the scope of this paper.

\section{OPTIMIZATION OF ANYONIC ENGINES: SHORTCUTS TO ADIABATICITY}

As we saw in the previous section, nonadiabatic driving significantly hinders engine performance through the loss of energy to nonadiabatic excitations - an effect typically referred to as "quantum friction." To achieve completely frictionless strokes, the cycle driving must be fully adiabatic, requiring infinite time and leading to zero power output. This trade-off can be circumvented through the use of "shortcuts to adiabaticity" (STA). STA refer to a set of techniques that can produce the same final state of a system in finite time that it would have achieved under adiabatic driving [134]. There are numerous established techniques to achieve this, including counterdiabatic driving [135-138], dynamical invariants [139], inversion of scaling laws [140], the fast-forward approach [141-145], optimal protocols [146-149], and time rescaling [150,151]. For a recent review on the topic of shortcuts see Ref. [61]. In this section we examine implementing optimal protocol and fast-forward STA for harmonic anyonic systems in order to determine if the anyonic phase plays a role in shortcut design.

\section{A. Optimal protocol shortcut}

We first examine the optimal protocol shortcut using the phenomenological framework of linear response theory $[62,147]$. This shortcut is based on separating the total nonequilibrium work into two contributions, the quasistatic work and the excess work,

$$
\langle W\rangle=\left\langle W_{\mathrm{QS}}\right\rangle+\left\langle W_{\mathrm{ex}}\right\rangle .
$$

Here $\left\langle W_{\mathrm{QS}}\right\rangle$ corresponds to the work carried out were the process to be fully quasistatic, and $\left\langle W_{\mathrm{ex}}\right\rangle$ the work lost due to nonequilibrium excitations. It has been shown that there exist optimal protocols for which $\left\langle W_{\text {ex }}\right\rangle$ vanishes, leading to quasistatic performance in finite time. This is a true STA, as for protocols where excess work vanishes no nonadiabatic transitions between eigenstates occur [147].

Let us consider a process in which we begin with a quantum system in thermal equilibrium with a reservoir at inverse temperature $\beta$. The system is then decoupled from the reservoir and driven by Hamiltonian $H(t) \equiv H\left(\lambda_{t}\right)$ 
where $\lambda_{t}$ is a time-dependent external control parameter, $\lambda_{t} \equiv \lambda_{0}+\delta \lambda g(t)$. This process corresponds exactly to the isentropic strokes of the harmonic Otto engine, with $\omega_{t}^{2}$ as the external control parameter.

If the external driving can be considered a weak perturbation, we can derive an expression for the excess work entirely from the equilibrium thermodynamic properties of the system using the tools of linear response theory. In this framework the excess work is given by [147]

$$
\left\langle W_{\mathrm{ex}}\right\rangle=-(\delta \lambda)^{2} \int_{t_{0}}^{t_{f}} d t \frac{\partial}{\partial t} g(t) \int_{0}^{t-t_{0}} d s \mathcal{R}(s) \frac{\partial}{\partial s} g(t-s),
$$

where $\mathcal{R}(t)$ is the relaxation function. Note that typically the relaxation function is denoted by $\Psi(t)$, as in Refs. [62,147]. Here we use $\mathcal{R}(t)$ to avoid confusion with the wave function. The relaxation function is determined by the quantum response function,

$$
\phi(t)=-\frac{\partial}{\partial t} \mathcal{R}(t)
$$

which is in turn found from the equilibrium state,

$$
\phi(t)=\frac{1}{i \hbar} \operatorname{tr}\left\{\rho_{0}\left[A_{0}, A_{t}\right]\right\} .
$$

Here $A_{t}$ is the generalized force,

$$
A=\frac{\partial}{\partial \lambda} H(\lambda)
$$

Let us first determine the excess work for two bosons and two fermions in one dimension. With these results we will be able to construct the excess work for one-dimensional statistical anyons. From the Hamiltonian in Eq. (25) we determine the time-dependent generalized force,

$$
A_{t}=\frac{1}{2} m\left[x_{1}^{2}(t)+x_{2}^{2}(t)\right]
$$

With Heisenberg's equation of motion and some elementary commutator algebra we determine $x(t)$, and from there the commutator $\left[A_{0}, A_{t}\right]$. Plugging this commutator into Eq. (58) we can then take the trace. To simplify the necessary integrals we first convert the thermal density operator to its Wigner distribution representation. The full expression for the Wigner distribution for bosons and fermions is given in Appendix C. In the Wigner distribution representation $x$ and $p$ are converted from operators to simple commuting variables. The resulting response functions are

$$
\phi(t)=\frac{\hbar}{4 \omega^{2}}[3 \operatorname{coth}(\beta \hbar \omega)+\operatorname{csch}(\beta \hbar \omega) \mp 1] \sin (2 t \omega),
$$

where the $(-)$ corresponds to bosons and the $(+)$ to fermions. From the response functions we can determine the relaxation functions with a trivial integral,

$$
\mathcal{R}(t)=\frac{\hbar}{8 \omega^{2}}[3 \operatorname{coth}(\beta \hbar \omega)+\operatorname{csch}(\beta \hbar \omega) \mp 1] \cos (2 t \omega) .
$$

We now have all the pieces we need to calculate the excess work from Eq. (56). For simplicity we take $t_{0}=0$ and $t_{f}=$ $\tau$. We pick a simple linear protocol $g(t)=t / \tau$, as it has been shown that there exist zeros of the excess work for a single particle in harmonic potential [147]. We find the excess work for two bosons or fermions to be

$$
\begin{aligned}
\left\langle W_{\mathrm{ex}}\right\rangle= & \frac{\hbar(\delta \omega)^{2}}{16 \omega_{0}^{5} \tau^{2}} \sin ^{2}\left(\omega_{0} \tau\right) \\
& \times\left[3 \operatorname{coth}\left(\beta \hbar \omega_{0}\right)+\operatorname{csch}\left(\beta \hbar \omega_{0}\right) \mp 1\right] .
\end{aligned}
$$

In Eqs. (50)-(52) we showed that, as the $N$ particle density operator is simply the product of the density operators of each individual particle pair, the statistical anyon internal energy is simply given by the weighted sum of the boson and fermion internal energies. The exact same mathematical process can be applied here in the calculation of the statistical anyon Wigner distribution, response function, relaxation function, and excess work. Thus we have

$$
\left\langle W_{\mathrm{ex}}^{\mathrm{SA}}\right\rangle=p_{B}\left\langle W_{\mathrm{ex}}^{B}\right\rangle+\left(1-p_{B}\right)\left\langle W_{\mathrm{ex}}^{F}\right\rangle .
$$

The statistical anyon excess work is plotted as a function of $\tau$ in Fig. 14. We see first that excess work varies with the anyonic phase, with fermions having the greatest excess work and bosons the least. We note that its zeros, however, are independent of the statistics. This is clear when examining Eq. (63). The statistics only come into play in the

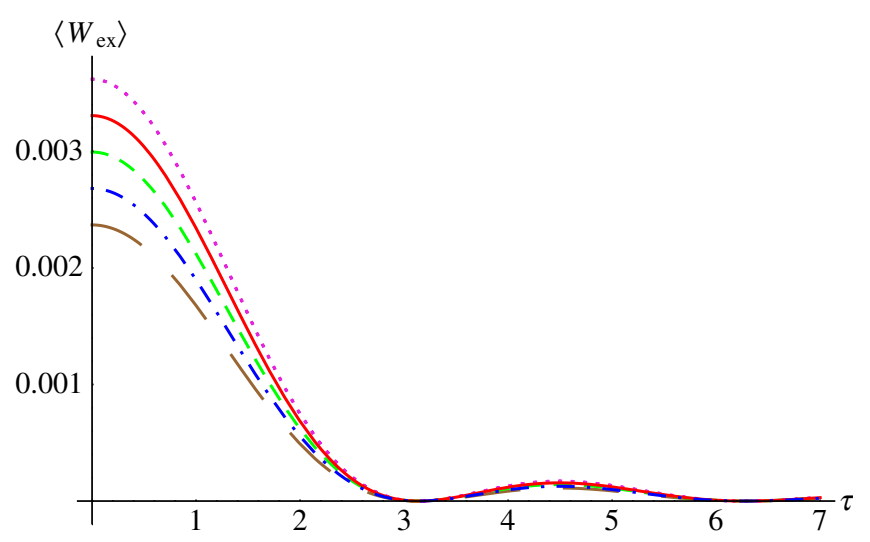

FIG. 14. Excess work under linear driving as a function of the driving time for two statistical anyons with $p_{B}=1$ (long dashed, brown), $p_{B}=0$ (dotted, dark pink), $p_{B}=1 / 2$ (dashed, green), $p_{B}=3 / 4$ (dot dashed, blue), and $p_{B}=1 / 4$ (solid, red) using a linear protocol. Parameters are $\omega_{0}=\beta=\hbar=1$, and $\delta \omega=0.1$. 


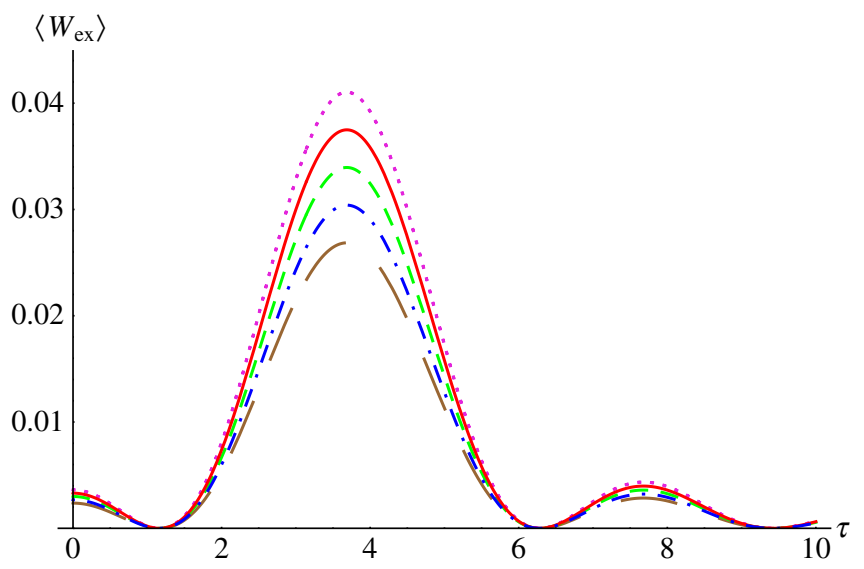

FIG. 15. Excess work under shortcut protocol driving as a function of the driving time for two statistical anyons with $p_{B}=1$ (long dashed, brown), $p_{B}=0$ (dotted, dark pink), $p_{B}=$ $1 / 2$ (dashed, green), $p_{B}=3 / 4$ (dot dashed, blue), and $p_{B}=1 / 4$ (solid, red) using the shortcut protocol. Parameters are $\omega_{0}=\beta=$ $\hbar=1, \alpha=1, \kappa=2$ and $\delta \omega=0.1$.

form of a $\mp 1$ within a multiplicative factor, which has no bearing on the zeros of the function. We see that for a linear protocol the one-dimensional statistical anyon excess work will vanish for all values of the anyonic phase when $\tau=n \pi / \omega$, where $n \in \mathbb{Z}$.

In Ref. [147] it was also shown that there exists a family of degenerate shortcut protocols that produce zeros of the excess work for a single particle in a parametric harmonic potential. This family take the form of

$$
g(t)=\frac{t}{\tau}+\alpha \sin (\kappa \pi t / \tau)
$$

where $\kappa \in \mathbb{Z}$ and $\alpha \in \mathbb{R}$. The excess work from this protocol is shown for one-dimensional statistical anyons in Fig. 15. We arrive at the same conclusion as in the case of the linear protocol. While the excess work itself depends on the statistics, the optimal shortcut protocol does not. Examining Eq. (56) we can extend this conclusion to any arbitrary protocol for the parametric harmonic potential. Since the factor in the expression for the excess work by which the bosons and fermions differ is independent of time, it will be identical for any $g(t)$.

\section{B. Fast-forward shortcut}

Having shown that the optimal protocol for achieving a shortcut to adiabaticity for one-dimensional statistical anyons in a harmonic Otto engine is independent of the quantum statistics, we next turn to a different shortcut implementation, the fast-forward method. Unlike the optimal protocol, in which the shortcut is determined entirely from the form of the thermal state and system Hamiltonian, the fast-forward shortcut is constructed using the instantaneous eigenstates. Since these eigenstates depend explicitly on the anyonic phase, we expect this dependence to carry through to the shortcut. To apply this method we introduce an auxiliary potential to the Schrödinger equation,

$$
i \hbar \frac{\partial}{\partial t} \Psi(\mathbf{x}, t)=-\frac{\hbar^{2}}{2 m} \nabla^{2} \Psi(\mathbf{x}, t)+\left(V+V_{\text {aux }}\right) \Psi(\mathbf{x}, t),
$$

where the form of $V_{\text {aux }}$ ensures the final state of the system after a finite time evolution is identical to that achieved after an adiabatic evolution of the unperturbed system. Let us consider the following ansatz for our time-dependent wave function:

$$
\Psi(\mathbf{x}, t)=\psi\left(\mathbf{x}, R_{t}\right) e^{i f(\mathbf{x}, t)} e^{-\frac{i}{\hbar} \int_{0}^{t} d s \epsilon\left(R_{s}\right)},
$$

where $\psi\left(\mathbf{x}, R_{t}\right)$ is the instantaneous eigenstate of the unperturbed Schrödinger equation with eigenenergy $\epsilon\left(R_{t}\right)$,

$$
\epsilon\left(R_{t}\right) \psi\left(\mathbf{x}, R_{t}\right)=-\frac{\hbar^{2}}{2 m} \nabla^{2} \psi\left(\mathbf{x}, R_{t}\right)+V \psi\left(\mathbf{x}, R_{t}\right) .
$$

Our goal is now to find the phase, $f(\mathbf{x}, t)$, and auxiliary potential $V_{\text {aux }}$ such that the above ansatz and equation are true. Imposing the condition that $f(\mathbf{x}, 0)=f(\mathbf{x}, \tau)$ (where $\tau$ is the duration of the driving) ensures that the final state is identical to that of the adiabatically driven unperturbed equation, and our shortcut is achieved.

To simplify the following analysis we express $\psi\left(\mathbf{x}, R_{t}\right)$ in polar representation:

$$
\psi\left(\mathbf{x}, R_{t}\right)=\mu\left(\mathbf{x}, R_{t}\right) e^{i \gamma\left(\mathbf{x}, R_{t}\right)} .
$$

Combining Eqs. (69), (67), and (66), simplifying, and separating the real and imaginary components we find

$$
V_{\text {aux }}=-\hbar \frac{\partial \gamma}{\partial t}-\hbar \frac{\partial f}{\partial t}-\frac{\hbar^{2}}{m} \nabla f \nabla \gamma-\frac{\hbar^{2}}{m}(\nabla f)^{2},
$$

and

$$
2 m \frac{\partial \mu}{\partial t}+2 \hbar \nabla f \nabla \mu+\hbar \mu \nabla^{2} f=0,
$$

where we write $\mu\left(\mathbf{x}, R_{t}\right)=\mu, \gamma\left(\mathbf{x}, R_{t}\right)=\gamma$, and $f(\mathbf{x}, t)=$ $f$ to simplify notation.

The shortcut method has a major caveat in that the auxiliary potential often becomes singular at the nodes of the instantaneous eigenstates, limiting its applicability to the ground state $[152,153]$. However, for the Lewis-Leach family of Hamiltonians, of which the harmonic oscillator is a member [154], it has been shown that the fast-forward potential is independent of the energy level $[143,152]$. 


\section{Statistical anyons}

As in the optimal protocol analysis let us first consider the fast-forward shortcut for the case of two bosons and two fermions, from which we will be able to construct the statistical anyon shortcut. It is straightforward to extend Eqs. (70) and (71) to a two-particle system,

$$
\begin{aligned}
V_{\text {aux }}= & -\hbar \frac{\partial \gamma}{\partial t}-\hbar \frac{\partial f}{\partial t}-\frac{\hbar^{2}}{m}\left(\nabla_{1} f \nabla_{1} \gamma+\nabla_{2} f \nabla_{2} \gamma\right) \\
& -\frac{\hbar^{2}}{m}\left[\left(\nabla_{1} f\right)^{2}+\left(\nabla_{2} f\right)^{2}\right], \\
2 m & \frac{\partial \mu}{\partial t}+2 \hbar\left(\nabla_{1} f \nabla_{1} \mu+\nabla_{2} f \nabla_{2} \mu\right) \\
& +\hbar \mu\left(\nabla_{1}^{2} f+\nabla_{2}^{2} f\right)=0 .
\end{aligned}
$$

As we seek shortcuts to optimize performance of our harmonic Otto engine let us again consider a one-dimensional harmonic potential. In this case we have $R(t)=\omega(t)$. We can express $\mu\left(x_{1}, x_{2}, \omega_{t}\right)$ for both bosons and fermions in terms of the single-particle harmonic oscillator eigenstates in the typical fashion,

$$
\begin{aligned}
\mu\left(x_{1}, x_{2}, \omega_{t}\right)= & \frac{1}{\sqrt{2\left(1+\delta_{n_{1}, n_{2}}\right)}}\left[\mu_{n_{1}}\left(x_{1}\right) \mu_{n_{2}}\left(x_{2}\right)\right. \\
& \left. \pm \mu_{n_{1}}\left(x_{2}\right) \mu_{n_{2}}\left(x_{1}\right)\right]
\end{aligned}
$$

where

$$
\mu_{n}(x)=\frac{1}{\sqrt{2^{n} n !}}\left(\frac{m \omega}{\pi \hbar}\right)^{1 / 4} e^{-\frac{m \omega x^{2}}{2 \hbar}} H_{n}\left(\sqrt{\frac{m \omega}{\hbar}} x\right)
$$

For a single particle in a harmonic potential Eq. (71) is solved by $[141,143]$

$$
f_{1}\left(x, \omega_{t}\right)=-\frac{m \dot{\omega}_{t}}{4 \pi \omega_{t}} x^{2} .
$$

With this in mind, we take our ansatz for the two-particle solution to be

$$
f_{2}\left(x_{1}, x_{2}, \omega_{t}\right)=-\frac{m \dot{\omega}_{t}}{4 \pi \omega_{t}}\left(x_{1}^{2}+x_{2}^{2}\right) .
$$

We can see that for any protocol with $\dot{\omega}_{0}=\dot{\omega}_{\tau}$ the shortcut condition will be fulfilled, as $f(\mathbf{x}, 0)=f(\mathbf{x}, \tau)$. A simple example of a protocol that fulfills this condition is the linear protocol, $\omega(t)=\omega_{0}+\alpha t$, where $\alpha$ is a constant.

With Eqs. (77) and (74) we can directly verify that our ansatz is correct by plugging in to Eq. (73). Since $\gamma\left(x_{1}, x_{2}, \omega_{t}\right)=0$ for both bosons and fermions, we can immediately see that not only the phase, but also the auxiliary potential will be identical for both bosons and fermions. Using Eq. (72) we determine the explicit form of the auxiliary potential to be

$$
V_{\mathrm{aux}}=-\frac{3 m \dot{\omega}_{t}}{8 \omega^{2}}\left(x_{1}^{2}+x_{2}^{2}\right) .
$$

Following the same method we can extend this analysis to two dimensions. For this case we take the ansatz,

$$
f_{2}\left(x_{1}, x_{2}, y_{1}, y_{2} \omega_{t}\right)=-\frac{m \dot{\omega}_{t}}{4 \pi \omega_{t}}\left(x_{1}^{2}+x_{2}^{2}+y_{1}^{2}+y_{2}^{2}\right),
$$

which we verify is a solution to Eq. (73). As in the one-dimensional case, $\gamma\left(x_{1}, x_{2}, y_{1}, y_{2}, \omega_{t}\right)=0$ so the auxiliary potential will again be identical for both bosons and fermions.

Since the statistical anyon state is constructed from a statistical average over the boson and fermion states, we know that if there is no difference in the shortcut for bosons and fermions, there will be none for statistical anyons. We conclude that in both one and two dimensions the implementation of a fast-forward STA is independent of the quantum statistics. This result is counter to our original hypothesis that the difference in phase of the instantaneous eigenstates should carry through to the design of the auxiliary potential. From the form of Eqs. (77) and (79) we can see that, physically, the two-particle shortcut for the harmonic oscillator potential is accomplished by driving each particle individually.

\section{Topological anyons}

Our previous analysis has shown that the behavior of topological anyons can have a richer dependence on the anyonic phase. Furthermore, topological anyon eigenstates are significantly more complex, and unlike bosonic or fermionic states can not generally be separated into a superposition of the single-particle states [43]. As the fastforward shortcut depends on the stationary eigenstate of the system it is of interest to explore whether or not a shortcut for topological anyons will show dependence on the anyonic phase.

Let us again consider the situation of two anyons in a harmonic potential. The time-independent Schrödinger equation for this problem can be solved by separating the Hamiltonian in Eq. (25) into the center of mass and relative components $[43,51,56]$,

$$
H=\hbar \omega\left(-\frac{\partial^{2}}{\partial Z \partial Z^{*}}-4 \frac{\partial^{2}}{\partial z \partial z^{*}}+|Z|^{2}+\frac{|z|^{2}}{4}\right),
$$

where we use the complex coordinates $z_{j}=\sqrt{m w / \hbar}\left(x_{j}+\right.$ $\left.i y_{j}\right)$ with $j \in 1,2$. Here $Z$ is the center-of-mass coordinate, given by $Z=\left(z_{1}+z_{2}\right) / 2$ and $z$ the relative coordinate, given by $z=z_{1}-z_{2}$. In two dimensions the anyonic exchange symmetry is satisfied by two separate families of 
eigenstates. The harmonic oscillator ground states for each are [51]

$$
\begin{aligned}
& \mu_{g}^{(I)}=A_{\nu} \omega z^{v} e^{-|Z|^{2}-\frac{|z|^{2}}{4}}, \\
& \mu_{g}^{(I I)}=A_{\nu} \omega\left(z^{*}\right)^{2-v} e^{-|Z|^{2}-\frac{|z|^{2}}{4}},
\end{aligned}
$$

where $A_{v}$ is an anyonic phase-dependent normalization factor. We can construct any desired excited state from these ground states by applying the appropriate raising operators [51].

In order to determine the fast-forward shortcut for the topological anyon system it will be convenient to convert Eqs. (72) and (73) into the center-of-mass and relative coordinate systems,

$$
\begin{aligned}
V_{\mathrm{aux}}=- & \frac{1}{\omega} \frac{\partial \gamma}{\partial t}-\frac{1}{\omega} \frac{\partial f}{\partial t}-\frac{\partial f}{\partial Z} \frac{\partial \gamma}{\partial Z^{*}}-\frac{\partial \gamma}{\partial Z} \frac{\partial f}{\partial Z^{*}}-\frac{\partial f}{\partial Z} \frac{\partial f}{\partial Z^{*}} \\
& -4 \frac{\partial f}{\partial z} \frac{\partial \gamma}{\partial z^{*}}-4 \frac{\partial \gamma}{\partial z} \frac{\partial f}{\partial z^{*}}-4 \frac{\partial f}{\partial z} \frac{\partial f}{\partial z^{*}} \\
\frac{\partial \mu}{\partial t}+ & \omega\left[\frac{\partial f}{\partial Z} \frac{\partial \mu}{\partial Z^{*}}+\frac{\partial \mu}{\partial Z} \frac{\partial f}{\partial Z^{*}}+\mu \frac{\partial^{2} f}{\partial Z \partial Z^{*}}\right. \\
& \left.+4 \frac{\partial f}{\partial z} \frac{\partial \mu}{\partial z^{*}}+4 \frac{\partial \mu}{\partial z} \frac{\partial f}{\partial z^{*}}+4 \beta \frac{\partial f}{\partial z \partial z^{*}}\right]=0
\end{aligned}
$$

Motivated by our results for the statistical anyons, we choose the same ansatz for $f$ (converted into the relative and center-of-mass coordinate system),

$$
f\left(Z, z, \omega_{t}\right)=-\frac{\dot{\omega}_{t}}{4 \omega^{2}}\left(\frac{1}{2}|z|^{2}+2|Z|^{2}\right) .
$$

Plugging Eqs. (84) and (81) into Eq. (83) we see that our ansatz is indeed a solution to the differential equation. As before, we have $\gamma\left(Z, z, \omega_{t}\right)=0$ in the center-of-mass and relative coordinate system. Using Eq. (82) we can construct the auxiliary potential,

$$
V_{\mathrm{aux}}=\frac{\dot{\omega}_{t}^{3}}{2 \omega^{4}}\left(\frac{1}{2}|z|^{2}+2|Z|^{2}\right) .
$$

We see that, as in the case of the statistical anyons, the fastforward STA for topological anyons is independent of the anyonic phase. This indicates that, counter to our original intuition, the harmonic oscillator fast-forward shortcut is truly independent of any exchange behavior, not just the bosonic and fermionic limits.

While this result was derived for the ground state we know that, since the harmonic oscillator Hamiltonian is a member of the Lewis-Leach family [154], the above shortcut will also hold for any excited state. This provides a physical motivation for why the shortcut is independent of the anyonic nature of the particles. Both the modified hard-core nature of topological anyons and the generalized exclusion statistics of statistical anyons affect how the particles will be distributed among the available states. Since the same harmonic oscillator shortcut holds for all states, it makes sense that it will be independent of the anyonic phase.

\section{CONCLUDING REMARKS}

\section{A. Summary}

In this work we have presented a dimensionindependent formulation of anyons, dubbed "statistical anyons," in which anyonic properties arise from averaging over the behavior of a system consisting of a statistical mixture of particles with antisymmetric and symmetric exchange properties. Motivated by the HOM effect, we outlined a quantum optics implementation of statistical anyons. We showed that the statistical anyons are physically equivalent to Haldane's generalized exclusion statistics anyons, broadening the applicability of GES to any system of indistinguishable particles for which such a mixture can be constructed.

We determined the thermodynamic properties of statistical anyons in one and two dimensions, and compared them to the thermodynamic properties of topological anyons. We found that in two dimensions the internal energy, free energy, entropy, and heat capacity all display a dependence on the anyonic phase, but it is not as rich a dependence as the topological anyons. However, we determined that, with a parameter-dependent choice of the anyonic phase, statistical anyons can exactly imitate the thermodynamic behavior of topological anyons.

With the thermodynamic properties established, we considered a harmonic quantum Otto engine with a working medium of statistical anyons. We found that in two dimensions endoreversible engine performance depends on the anyonic phase for both statistical anyons and topological anyons. We found that for the nonequilibrium regime, even in one dimension, the engine EMP for statistical anyons depended on the anyonic phase.

Lastly, we examined the role of the anyonic phase in two STA, the optimal protocol shortcut and the fast-forward shortcut. We found both shortcut methods are independent of the anyonic nature of the particles. In the case of the fast-forward method, this independence can be considered to arise from the fact that the shortcut does not depend on the energy level for a harmonic potential.

\section{B. Impacts and future directions}

In this work we have taken a device-oriented approach for examining the thermodynamic properties of anyons, using the framework of heat engines. This method is motivated by the history of thermodynamics, as a field 
developed around the optimization of thermal machines, and is an approach commonly used in modern quantum thermodynamics [60]. To the authors' knowledge, this approach has only been applied in two previous works, both based around Calogero-Sutherland GES anyons [105, 119]. However, we believe this work is the first to analyze heat engines and shortcuts for topological anyons, and to make an explicit comparison between both the GES and topological implementations of anyons in this context.

While functionally equivalent to GES, statistical anyons present a new paradigm both theoretically and experimentally that extends GES to a range of new settings, including Bose-Fermi mixtures [90-95], optimechanical systems [155], and surface plasmonics [156]. While they lack the non-Abelian properties necessary for implementing topological quantum computation, they do provide an experimentally tractable method of examining the thermodynamic behavior of Abelian topological anyons. This opens up the door to searching for general thermodynamic signatures that may provide alternative methods of detecting and controlling both Abelian and non-Abelian anyons.

Using statistical anyons we have connected topics across various subfields of physics, including the Hong-OuMandel effect from quantum optics, heat engines from quantum thermodynamics, GES from quantum statistical mechanics, fractional exchange statistics from topological states of matter, and shortcuts to adiabaticity from quantum control. The establishment of the statistical anyon framework opens up a multitude of new possible research directions and questions for the study of anyons. There remains much to be explored about the thermodynamics of statistical anyons, including Gibbs mixing for statistical anyons [157] and the behavior of autonomous quantum engines with statistical anyon working mediums. We have shown in this work that the performance of a cyclic quantum engine is sensitive to the anyonic phase, and we would expect the same to be true for autonomous engines. Implementing the latter in an optical or plasmonic setting has metrological implications as an alternative device for detecting signatures of anyonic behavior.

It would be of interest to compare statistical anyons to other methods of generating anyonic behavior not covered in this work, such as N00N states subject to Bloch oscillations [158], quasiholes in Bose-Einstein condensates [159], or particles possessing ambiguous statistics [160]. Statistical anyons may also have the potential to provide simpler implementations or experimental analogs for other applications of anyons, including Haldane insulators $[161,162]$ or anyon beams [163]. Anyonic statistics has also been applied to more exotic systems, such as blackhole gasses $[160,164]$, for which statistical anyons may provide a tractable theoretical tool. Finally, a particularly interesting possibility for statistical anyons is the introduction of interparticle interactions. We have shown that noninteracting statistical anyons are capable of recreating GES, which historically has been limited to interacting systems. By combining these paradigms, we add significant complexity to statistical behavior of the particles. It is intriguing to wonder if this additional complexity may be leveraged to expand the state space of possible anyonic phases, effectively recreating the braiding statistics of topological anyons. We leave this multitude of questions open for exploration in future works.

\section{ACKNOWLEDGMENTS}

S.D. acknowledges support from the U.S. National Science Foundation under Grant No. DMR-2010127.

\section{APPENDIX A: STATISTICAL ANYON HARMONIC OSCILLATOR PARTITION FUNCTION}

In this Appendix we derive an expression for the partition function of two statistical anyons in a harmonic potential. We begin with the definition of the partition function for $N$-independent pairs of particles in the position basis,

$$
Z_{A}^{N}=\operatorname{tr}\left\{e^{-\beta H}\right\}=\prod_{j=1}^{N} \int d x_{j} \int d y_{j}\left\langle x_{j} y_{j}\left|e^{-\beta H_{j}}\right| x_{j} y_{j}\right\rangle
$$

where

$$
H_{j}=\frac{p_{x_{j}}^{2}+p_{y_{j}}^{2}}{2 m}+\frac{1}{2} m \omega^{2}\left(x_{j}^{2}+y_{j}^{2}\right)
$$

Inserting the identity in the energy basis $I=\sum_{n_{1}^{(j)}, n_{2}^{(j)}=0}^{\infty}$ $\left|n_{1}^{(j)} n_{2}^{(j)}\right\rangle\left\langle n_{1}^{(j)} n_{2}^{(j)}\right|$ twice leads to

$$
\begin{aligned}
Z_{A}^{N}= & \prod_{j=1}^{N} \int d x_{j} \int d y_{j} \sum_{n_{1}^{(j)}, n_{2}^{(j)}=0}^{\infty} \sum_{m_{1}^{(j)}, m_{2}^{(j)}=0}^{\infty} \Psi_{A}^{*}\left(x_{j}, y_{j}\right) \\
& \times \Psi_{A}\left(x_{j}, y_{j}\right) e^{-\beta \hbar \omega\left(m_{1}^{(j)}+m_{2}^{(j)}+1\right)} \\
& \times\left\langle n_{1}^{(j)} n_{2}^{(j)} \mid m_{1}^{(j)} m_{2}^{(j)}\right\rangle .
\end{aligned}
$$

Here $\Psi_{A}\left(x_{j}, y_{j}\right)$ is given by Eq. (14), where

$$
\psi_{n}(x)=\frac{1}{\sqrt{2^{n} n !}}\left(\frac{m \omega}{\pi \hbar}\right)^{1 / 4} e^{-\frac{m \omega x^{2}}{2 \hbar}} H_{n}\left(\sqrt{\frac{m \omega}{\hbar}} x\right)
$$

After evaluating the inner product, the integration over each $x_{j}$ and $y_{j}$ can be carried out with application of the 
Hermite polynomial orthogonality to yield

$$
\begin{aligned}
Z_{A}^{N}= & \prod_{j=1}^{N} \sum_{n_{1}^{(j)}, n_{2}^{(j)}=0}^{\infty} \frac{1}{4} e^{-\beta \hbar \omega\left(n_{1}^{(j)}+n_{2}^{(j)}+1\right)} \\
& \times\left(2+e^{-i \pi \Theta\left(j-N_{B}+1\right)} \delta_{n_{1}^{(j)}, n_{2}^{(j)}}\right. \\
& \left.+e^{i \pi \Theta\left(j-N_{B}+1\right)} \delta_{n_{1}^{(j)}, n_{2}^{(j)}}\right),
\end{aligned}
$$

where $\delta_{n_{1}, n_{2}}$ is the Kronecker delta. In order to evaluate the step functions we separate the product into one from 1 to $N_{B}$ and a second from $N_{B}+1$ to $N$,

$$
\begin{aligned}
Z_{A}^{N}= & \prod_{j=1}^{N_{B}} \sum_{n_{1}^{(j)}, n_{2}^{(j)}=0}^{\infty} \frac{1}{2} e^{-\beta \hbar \omega\left(n_{1}^{(j)}+n_{2}^{(j)}+1\right)}\left(1+\delta_{n_{1}^{(j)}, n_{2}^{(j)}}\right) \\
& \prod_{j=N_{B}+1}^{N} \sum_{n_{1}^{(j)}, n_{2}^{(j)}=0}^{\infty} \frac{1}{2} e^{-\beta \hbar \omega\left(n_{1}^{(j)}+n_{2}^{(j)}+1\right)}\left(1-\delta_{n_{1}^{(j)}, n_{2}^{(j)}}\right) .
\end{aligned}
$$

Here we can immediately recognize the individual partition functions for two bosons and two fermions in harmonic potential,

$$
\begin{aligned}
& Z_{B}=\sum_{n_{1}, n_{2}=0}^{\infty} \frac{1}{2}\left(1+\delta_{n_{1}, n_{2}}\right) e^{-\beta \hbar \omega\left(n_{1}+n_{2}+1\right)}, \\
& Z_{F}=\sum_{n_{1}, n_{2}=0}^{\infty} \frac{1}{2}\left(1-\delta_{n_{1}, n_{2}}\right) e^{-\beta \hbar \omega\left(n_{1}+n_{2}+1\right)} .
\end{aligned}
$$

Thus we can rewrite our anyonic partition function in the simple form,

$$
Z_{A}^{N}=\prod_{j=1}^{N_{B}} Z_{B}^{(j)} \prod_{k=1}^{N_{F}} Z_{F}^{(k)}=Z_{B}^{N_{B}} Z_{F}^{N_{F}}=\left(Z_{B}^{p_{B}} Z_{F}^{p_{F}}\right)^{N} .
$$

Here we use the fact that for large $N$ we can express the number of bosonic and fermionic particle pairs as $N_{B}=$ $N p_{B}$ and $N_{F}=N p_{F}=N\left(1-p_{B}\right)$ where $p_{B}\left(p_{F}\right)$ is the probability of a particle pair having bosonic (fermionic) symmetry.

The boson and fermion partition functions can be written in closed form as

$$
\begin{aligned}
& Z_{B}=\frac{1}{8} \operatorname{csch}^{2}\left(\frac{\beta \omega \hbar}{2}\right)+\frac{1}{4} \operatorname{csch}(\beta \omega \hbar), \\
& Z_{F}=\frac{1}{8} \operatorname{csch}^{2}\left(\frac{\beta \omega \hbar}{2}\right)-\frac{1}{4} \operatorname{csch}(\beta \omega \hbar) .
\end{aligned}
$$

With this, we arrive at a closed-form expression for the partition function of a single pair of statistical anyons,

$$
\begin{aligned}
Z_{A}= & {\left[\frac{1}{8} \operatorname{csch}^{2}\left(\frac{\beta \omega \hbar}{2}\right)+\frac{1}{4} \operatorname{csch}(\beta \omega \hbar)\right]^{p_{B}} } \\
& \times\left[\frac{1}{8} \operatorname{csch}^{2}\left(\frac{\beta \omega \hbar}{2}\right)-\frac{1}{4} \operatorname{csch}(\beta \omega \hbar)\right]^{1-p_{B}} .
\end{aligned}
$$

\section{APPENDIX B: EQUILIBRIUM THERMODYNAMIC QUANTITIES}

In this Appendix we give the full expressions for the internal energies, free energies, entropies, and heat capacities of statistical anyons and topological anyons in oneand two-dimensional harmonic potentials.

\section{2D statistical anyons}

Plugging the respective partition functions for two bosons and two fermions in a harmonic potential into Eq. (26), and substituting that into Eq. (27) we arrive at the following expressions for the thermodynamic properties of two-dimensional statistical anyons:

$$
\begin{aligned}
E= & \hbar \omega\left[2 \operatorname{coth}\left(\frac{1}{2} \beta \hbar \omega\right)+\tanh \left(\frac{1}{2} \beta \hbar \omega\right)-p_{B} \tanh (\beta \hbar \omega)\right] \\
F= & -\frac{1}{\beta} \ln \left\{\frac{1}{8}[\cosh (\beta \hbar \omega)]^{p_{B}} \operatorname{csch}^{2}\left(\frac{1}{2} \beta \hbar \omega\right) \operatorname{csch}^{2}(\beta \hbar \omega)\right\} \\
S= & k_{B} \beta \hbar \omega\left[2 \operatorname{coth}\left(\frac{1}{2} \beta \hbar \omega\right)+\tanh \left(\frac{1}{2} \beta \hbar \omega\right)-p_{B} \tanh (\beta \hbar \omega)\right] \\
& +k_{B} \ln \left\{\frac{1}{8}[\cosh (\beta \hbar \omega)]^{p_{B}} \operatorname{csch}^{2}\left(\frac{1}{2} \beta \hbar \omega\right) \operatorname{csch}^{2}(\beta \hbar \omega)\right\} \\
C= & \frac{1}{2} k_{B} \beta^{2} \hbar^{2} \omega^{2}\left[2 \operatorname{csch}^{2}\left(\frac{1}{2} \beta \hbar \omega\right)-\operatorname{sech}^{2}\left(\frac{1}{2} \beta \hbar \omega\right)+2 p_{B} \operatorname{sech}^{2}(\beta \hbar \omega)\right]
\end{aligned}
$$




\section{2D topological anyons}

Plugging Eq. (29) into Eq. (27) we determine the following expressions for the thermodynamic properties of topological anyons:

$$
\begin{aligned}
E= & \hbar \omega\left\{\operatorname{coth}\left(\frac{1}{2} \beta \hbar \omega\right)+2 \operatorname{coth}(\beta \hbar \omega)+(v-1) \tanh [\beta \hbar \omega(1-v)]\right\} \\
F= & \frac{1}{\beta} \ln \left\{8 \operatorname{sech}[\beta \hbar \omega(v-1)] \sinh ^{2}\left(\frac{1}{2} \beta \hbar \omega\right) \sinh ^{2}(\beta \hbar \omega)\right\} \\
S= & k_{B} \beta \hbar \omega\left\{\operatorname{coth}\left(\frac{1}{2} \beta \hbar \omega\right)+2 \operatorname{coth}(\beta \hbar \omega)+(v-1) \tanh [\beta \hbar \omega(1-v)]\right\} \\
& +k_{B} \ln \left\{\frac{1}{8} \cosh [\beta \hbar \omega(v-1)] \operatorname{csch}^{2}\left(\frac{1}{2} \beta \hbar \omega\right) \operatorname{csch}^{2}(\beta \hbar \omega)\right\} \\
C= & \frac{1}{2} k_{B} \beta^{2} \hbar^{2} \omega^{2}\left\{\operatorname{csch}^{2}\left(\frac{1}{2} \beta \hbar \omega\right)+4 \operatorname{csch}^{2}(\beta \hbar \omega)+2(v-1)^{2} \operatorname{sech}^{2}[\beta \hbar \omega(v-1)]\right\} .
\end{aligned}
$$

\section{APPENDIX C: 1D BOSON AND FERMION DENSITY OPERATORS AND WIGNER DISTRIBUTIONS}

In this Appendix we give the full expressions for the thermal state and time-evolved density operators for a nonequilibrium harmonic quantum Otto engine with a working medium of 1D bosons and fermions. We also include the thermal state Wigner distribution applied in the determination of the optimal protocol shortcut. In each expression the top sign of each plus and minus denotes the boson expression and the bottom sign the fermion expression.

The thermal-state density operator in position representation is given by

$$
\begin{aligned}
\rho_{0}\left(x_{1}, x_{2}, y_{1}, y_{2}\right)= & \frac{1}{Z} \frac{m \omega}{2 \pi \hbar \sinh (\beta \hbar \omega)} \\
& \times\left[e^{-\frac{m \omega}{4 \hbar}\left\{\left[\left(x_{1}+y_{1}\right)^{2}+\left(x_{2}+y_{2}\right)^{2}\right] \tanh (\beta \hbar \omega / 2)+\left[\left(x_{1}-y_{1}\right)^{2}+\left(x_{2}-y_{2}\right)^{2}\right] \operatorname{coth}(\beta \hbar \omega / 2)\right\}}\right. \\
& \left. \pm e^{-\frac{m \omega}{4 \hbar}\left\{\left[\left(x_{2}+y_{1}\right)^{2}+\left(x_{1}+y_{2}\right)^{2}\right] \tanh (\beta \hbar \omega / 2)+\left[\left(x_{2}-y_{1}\right)^{2}+\left(x_{1}-y_{2}\right)^{2}\right] \operatorname{coth}(\beta \hbar \omega / 2)\right\}}\right] .
\end{aligned}
$$

Solving the time-dependent Schrödinger equation by means of the appropriate evolution operator for the isentropic strokes of the engine yields the time-evolved density operator,

$$
\begin{aligned}
& \rho_{t}\left(x_{1}, x_{2}, y_{1}, y_{2}\right)=\frac{m \omega}{2 \pi \hbar\left(Y_{t}^{2}+X_{t}^{2} \omega^{2}\right)}\left(e^{\mp \beta \hbar \omega}-1\right) \\
& \times\left\{e^{\frac{m}{2 \hbar\left(Y_{t}^{2}+X_{t}^{2} \omega^{2}\right)}\left[i\left(x_{1}^{2}+x_{2}^{2}-y_{1}^{2}-y_{2}^{2}\right)\left(Y_{t} \dot{Y}_{t}+X_{t} \dot{X}_{t} \omega^{2}\right)-\omega\left(x_{1}^{2}+x_{2}^{2}+y_{1}^{2}+y_{2}^{2}\right) \operatorname{coth}(\beta \hbar \omega)+2 \omega\left(x_{1} y_{1}+x_{2} y_{2}\right) \operatorname{csch}(\beta \hbar \omega)\right]}\right. \\
& \left. \pm e^{\frac{m}{2 \hbar\left(Y_{t}^{2}+X_{t}^{2} \omega^{2}\right)}\left[i\left(x_{1}^{2}+x_{2}^{2}-y_{1}^{2}-y_{2}^{2}\right)\left(Y_{t} \dot{Y}_{t}+X_{t} \dot{X}_{t} \omega^{2}\right)-\omega\left(x_{1}^{2}+x_{2}^{2}+y_{1}^{2}+y_{2}^{2}\right) \operatorname{coth}(\beta \hbar \omega)+2 \omega\left(x_{2} y_{1}+x_{1} y_{2}\right) \operatorname{csch}(\beta \hbar \omega)\right]}\right\} \text {. }
\end{aligned}
$$

Here $X_{t}$ and $Y_{t}$ are solutions to the equation of motion of the classical time-dependent harmonic oscillator,

$$
\ddot{X}_{t}+\omega^{2}(t) X_{t}=0
$$

The two-particle Wigner distribution is found by carrying out the integral,

$$
W\left(x_{1}, p_{1}, x_{2}, p_{2}\right)=\frac{1}{4 \pi^{2} \hbar^{2}} \int d u_{1} \int d u_{2} \rho\left(x_{1}+\frac{u_{1}}{2}, x_{2}+\frac{u_{2}}{2}, x_{1}-\frac{u_{1}}{2}, x_{2}-\frac{u_{2}}{2}\right) e^{-\frac{i p_{1} u_{1}}{\hbar}} e^{-\frac{i p_{2} u_{2}}{\hbar}} .
$$


For the thermal state the Wigner distribution is

$$
\begin{aligned}
W_{0}\left(x_{1}, p_{1}, x_{2}, p_{2}\right)= & \frac{\operatorname{sech}^{2}(\beta \hbar \omega / 2)}{\pi^{2} \hbar^{2}\left[\operatorname{csch}^{2}(\beta \hbar \omega / 2) \pm 2 \operatorname{csch}(\beta \hbar \omega)\right]} \\
& \times\left(e^{-\frac{\left.p_{1}^{2}+p_{2}^{2}+m^{2}\left(x_{1}^{2}+x_{2}^{2}\right) \omega^{2}\right] \tanh (\beta \hbar \omega / 2)}{m \omega \hbar}} \pm 2 e^{\frac{-\left[p_{1}^{2}+p_{2}^{2}+m^{2}\left(x_{1}^{2}+x_{2}^{2}\right) \omega^{2}\right] \operatorname{coth}(\beta \hbar \omega)+2\left(p_{1} p_{2}+m^{2} \omega^{2} x_{1} x_{2}\right) \operatorname{csch}(\beta \hbar \omega)}{m \omega \hbar}}\right) .
\end{aligned}
$$

\section{APPENDIX D: NONEQUILIBRIUM ENGINE CHARACTERIZATIONS: 1D STATISTICAL ANYONS}

In this Appendix we give the full expressions for the power and efficiency of a nonequilibrium harmonic quantum Otto engine with a working medium of 1D statistical anyons.

$$
\begin{aligned}
& P= \frac{\hbar}{2 \tau}\left\{\left(2 p_{B}-1\right)\left[\left(Q_{21}-1\right) \omega_{1}+\left(Q_{12}-1\right) \omega_{2}\right]+3\left(\omega_{2}-Q_{21} \omega_{1}\right) \operatorname{coth}\left(\beta_{2} \omega_{2} \hbar\right)\right. \\
&\left.+3\left(\omega_{1}-Q_{12} \omega_{2}\right) \operatorname{coth}\left(\beta_{1} \omega_{1} \hbar\right)+\left(\omega_{2}-Q_{21} \omega_{1}\right) \operatorname{csch}\left(\beta_{2} \omega_{2} \hbar\right)+\left(\omega_{1}-Q_{12} \omega_{2}\right) \operatorname{csch}\left(\beta_{1} \omega_{1} \hbar\right)\right\} \\
& \eta=1+\frac{\omega_{1}}{\omega_{2}}\left\{\frac{3 \operatorname{coth}\left(\beta_{1} \omega_{1} \hbar\right)+\operatorname{csch}\left(\beta_{1} \omega_{1} \hbar\right)+2\left(Q_{21}-1\right) p_{B}-Q_{21}\left[3 \operatorname{coth}\left(\beta_{2} \omega_{2} \hbar\right)+\operatorname{csch}\left(\beta_{2} \omega_{2} \hbar\right)+1\right]+1}{3 \operatorname{coth}\left(\beta_{2} \omega_{2} \hbar\right)+\operatorname{csch}\left(\beta_{2} \omega_{2} \hbar\right)+2\left(Q_{12}-1\right) p_{B}-Q_{12}\left[3 \operatorname{coth}\left(\beta_{1} \omega_{1} \hbar\right)+\operatorname{csch}\left(\beta_{1} \omega_{1} \hbar\right)+1\right]+1}\right\} .
\end{aligned}
$$

[1] D. J. Griffiths, Introduction to Quantum Mechanics (Cambridge University Press, New York, 2017).

[2] J. M. Leinaas and J. Myrheim, On the theory of identical particles, Nuovo Cim. B 37, 1 (1977).

[3] F. Wilczek, Quantum Mechanics of Fractional-Spin Particles, Phys. Rev. Lett. 49, 957 (1982).

[4] D. Arovas, J. R. Schrieffer, and F. Wilczek, Fractional Statistics and the Quantum Hall Effect, Phys. Rev. Lett. 53, 722 (1984).

[5] G. Moore and N. Read, Nonabelions in the fractional quantum hall effect, Nucl. Phys. B 360, 362 (1991).

[6] A. Kitaev, Fault-tolerant quantum computation by anyons, Ann. Phys. 303, 2 (2003).

[7] D. J. Clarke, J. Alicea, and K. Shtengel, Exotic nonAbelian anyons from conventional fractional quantum Hall states, Nat. Comm. 4, 1348 (2013).

[8] Y. Kasahara, T. Ohnishi, Y. Mizukami, O. Tanaka, S. Ma, K. Sugii, N. Kurita, H. Tanaka, J. Nasu, Y. Motome, T. Shibauchi, and Y. Matsuda, Majorana quantization and half-integer thermal quantum Hall effect in a Kitaev spin liquid, Nature 559, 227 (2018).

[9] M. Banerjee, M. Heiblum, V. Umansky, D. E. Feldman, Y. Oreg, and A. Stern, Observation of half-integer thermal Hall conductance, Nature 559, 205 (2018).

[10] C. Nayak, S. H. Simon, A. Stern, M. Freedman, and S. Das Sarma, Non-Abelian anyons and topological quantum computation, Rev. Mod. Phys. 80, 1083 (2008).

[11] F. D. M. Haldane, Fractional Statistics in Arbitrary Dimensions: A Generalization of the Pauli Principle, Phys. Rev. Lett. 67, 937 (1991).
[12] M. V. N. Murthy and R. Shankar, Haldane Exclusion Statistics and Second Virial Coefficient, Phys. Rev. Lett. 72, 3629 (1994).

[13] Y.-S. Wu, Statistical Distribution for Generalized Ideal Gas of Fractional-Statistics Particles, Phys. Rev. Lett. 73, 922 (1994).

[14] M. V. N. Murthy and R. Shankar, Thermodynamics of a One-Dimensional Ideal Gas with Fractional Exclusion Statistics, Phys. Rev. Lett. 73, 3331 (1994).

[15] A. Dasnières de Veigy and S. Ouvry, One-dimensional statistical mechanics for identical particles: The calogero and anyon cases, Mod. Phys. Lett. B 09, 271 (1995).

[16] A. Bytsko and A. Fring, Thermodynamic bethe ansatz with Haldane statistics, Nuclear Phys. B 532, 588 (1998).

[17] S. Meljanac, M. Mileković, and A. Samsarov, Generalized calogero model in arbitrary dimensions, Phys. Lett. B 594, 241 (2004).

[18] A. Comtet, S. N. Majumdar, and S. Ouvry, Integer partitions and exclusion statistics, J. Phys. A Math. Theor. 40, 11255 (2007).

[19] A. del Campo, Exact quantum decay of an interacting many-particle system: The calogero-sutherland model, New J. Phys. 18, 015014 (2016).

[20] E. H. Lieb and W. Liniger, Exact analysis of an interacting bose gas. I. The general solution and the ground state, Phys. Rev. 130, 1605 (1963).

[21] C. N. Yang and C. P. Yang, Thermodynamics of a one-dimensional system of bosons with repulsive deltafunction interaction, J. Math. Phys. 10, 1115 (1969).

[22] C. P. Yang, One-dimensional system of bosons with repulsive $\delta$-function interactions at a finite temperature $t$, Phys. Rev. A 2, 154 (1970). 
[23] D. Bernard and Y.-S. Wu, A note on statistical interactions and the thermodynamic Bethe ansatz, arXiv:cond-mat/9404025 (1994).

[24] S. B. Isakov, D. P. Arovas, J. Myrheim, and A. P. Polychronakos, Thermodynamics for fractional exclusion statistics, Phys. Lett. A 212, 299 (1996).

[25] C. Aneziris, A. Balachandran, and D. Sen, Statistics in one dimension, Int. J. Mod. Phys. A 06, 4721 (1991).

[26] M. T. Batchelor and X.-W. Guan, Fermionization and fractional statistics in the strongly interacting one-dimensional bose gas, Laser Phys. Lett. 4, 77 (2007).

[27] S. Ouvry and A. P. Polychronakos, The Lieb-Liniger model in the infinite coupling constant limit, J. Phys. A Math 42, 275302 (2009).

[28] R. K. Bhaduri, S. M. Reimann, S. Viefers, A. G. Choudhury, and M. K. Srivastava, The effect of interactions on bose-einstein condensation in a quasi two-dimensional harmonic trap, J Phys. B: At. Mol. Opt. 33, 3895 (2000).

[29] S. Viefers, T. H. Hansson, and J. M. Leinaas, Exclusion statistics in a two-dimensional trapped bose gas, J Phys. B: At. Mol. Opt. 34, 4609 (2001).

[30] A. Kundu, Exact Solution of Double $\delta$ Function Bose Gas through an Interacting Anyon Gas, Phys. Rev. Lett. 83, 1275 (1999).

[31] M. T. Batchelor, X.-W. Guan, and N. Oelkers, OneDimensional Interacting Anyon Gas: Low-Energy Properties and Haldane Exclusion Statistics, Phys. Rev. Lett. 96, 210402 (2006).

[32] M. T. Batchelor and X.-W. Guan, Generalized exclusion statistics and degenerate signature of strongly interacting anyons, Phys. Rev. B 74, 195121 (2006).

[33] M. T. Batchelor, X.-W. Guan, and J.-S. He, The Bethe ansatz for 1D interacting anyons, J. Stat. Mech.: Theory Exp. 2007, P03007 (2007).

[34] P. Calabrese and M. Mintchev, Correlation functions of one-dimensional anyonic fluids, Phys. Rev. B 75, 233104 (2007).

[35] O. I. Pâţu, V. E. Korepin, and D. V. Averin, Correlation functions of one-dimensional Lieb-Liniger anyons, J. Phys. A 40, 14963 (2007).

[36] M. T. Batchelor, X.-W. Guan, and A. Kundu, Onedimensional anyons with competing $\delta$-function and derivative $\delta$-function potentials, J. Phys. A 41, 352002 (2008).

[37] Y. Hao, Y. Zhang, and S. Chen, Ground-state properties of one-dimensional anyon gases, Phys. Rev. A 78, 023631 (2008).

[38] A. del Campo, Fermionization and bosonization of expanding one-dimensional anyonic fluids, Phys. Rev. A 78, 045602 (2008).

[39] O. I. Pâţu, V. E. Korepin, and D. V. Averin, Onedimensional impenetrable anyons in thermal equilibrium: I. Anyonic generalization of Lenard's formula, J. Phys. A 41, 145006 (2008).

[40] O. I. Pâţu, V. E. Korepin, and D. V. Averin, Onedimensional impenetrable anyons in thermal equilibrium: II. Determinant representation for the dynamic correlation functions, J. Phys. A 41, 255205 (2008).

[41] R. Santachiara and P. Calabrese, One-particle density matrix and momentum distribution function of one-dimensional anyon gases, J. Stat. Mech.: Theory Exp. 2008, P06005 (2008).

[42] S. B. Isakov, Statistical Mechanics for a Class of Quantum Statistics, Phys. Rev. Lett. 73, 2150 (1994).

[43] A. Khare, Fractional Statistics and Quantum Theory (World Scientific, Singapore, 2005), 2nd ed.

[44] A. Rovenchak, Two-parametric fractional statistics models for anyons, Euro. Phys.1 J. B 87, 175 (2014).

[45] G. S. Joyce, S. Sarkar, J. Spalek, and K. Byczuk, Thermodynamic properties of particles with intermediate statistics, Phys. Rev. B 53, 990 (1996).

[46] D.-V. Anghel, From fractional exclusion statistics back to Bose and Fermi distributions, Phys. Lett. A 377, 2922 (2013).

[47] S. Ouvry and A. P. Polychronakos, Exclusion statistics for particles with a discrete spectrum, arXiv:2105.14042 (2021).

[48] S. B. Isakov, Fractional statistics in one dimension: Modeling by means of $1 / x^{2}$ interaction and statistical mechanics, Int. J. Mod. Phys. A 09, 2563 (1994).

[49] X.-W. Guan and Y.-Y. Chen, in 60 Years of Yang-Mills Gauge Field Theories (2016), p. 399.

[50] R. K. Bhaduri, R. S. Bhalerao, A. Khare, J. Law, and M. V. N. Murthy, Semiclassical two- and Three-Anyon Partition Functions, Phys. Rev. Lett. 66, 523 (1991).

[51] J. Myrheim, in Topological aspects of low dimensional systems, edited by A. Comtet, T. Jolicœur, S. Ouvry, and F. David (Springer, Berlin, 1999) p. 265.

[52] D. P. Arovas, R. Schrieffer, F. Wilczek, and A. Zee, Statistical mechanics of anyons, Nucl. Phys. B. 251, 117 (1985).

[53] D. Sen, Spectrum of Three Anyons in a Harmonic Potential and the Third Virial Coefficient, Phys. Rev. Lett. 68, 2977 (1992).

[54] M. Sporre, J. Verbaarschot, and I. Zahed, Anyon spectra and the third virial coefficient, Nucl. Phys. B 389, 645 (1993).

[55] P. Giacconi, F. Maltoni, and R. Soldati, Second virial coefficient for contact-interacting anyons, Phys. Rev. B 53, 10065 (1996).

[56] A. Lerda, Anyons: Quantum Mechanics of Particles with Fractional Statistics (Springer, Berlin, 1992).

[57] W. Chen, Thermodynamics of an anyon system, Phys. Rev. D 52, 6122 (1995).

[58] J. Nasu and Y. Motome, Thermodynamics of Chiral Spin Liquids with Abelian and Non-Abelian Anyons, Phys. Rev. Lett. 115, 087203 (2015).

[59] A. P. Polychronakos, Virial Coefficients of non-Abelian Anyons, Phys. Rev. Lett. 84, 1268 (2000).

[60] S. Deffner and S. Campbell, Quantum Thermodynamics (Morgan and Claypool, Bristol, 2019).

[61] D. Guéry-Odelin, A. Ruschhaupt, A. Kiely, E. Torrontegui, S. Martínez-Garaot, and J. G. Muga, Shortcuts to adiabaticity: Concepts, methods, and applications, Rev. Mod. Phys. 91, 045001 (2019).

[62] S. Deffner and M. V. S. Bonança, Thermodynamic control — an old paradigm with new applications, EPL 131, 20001 (2020).

[63] H. E. D. Scovil and E. O. Schulz-DuBois, Three-Level Masers as Heat Engines, Phys. Rev. Lett. 2, 262 (1959). 
[64] E. Köse, S. m. c. Çakmak, A. Gençten, I. K. Kominis, and O. E. Müstecaplğlu, Algorithmic quantum heat engines, Phys. Rev. E 100, 012109 (2019).

[65] C. de C. Chamon, D. E. Freed, S. A. Kivelson, S. L. Sondhi, and X. G. Wen, Two point-contact interferometer for quantum hall systems, Phys. Rev. B 55, 2331 (1997).

[66] Y. Ji, Y. Chung, D. Sprinzak, M. Heiblum, D. Mahalu, and H. Shtrikman, An electronic Mach-Zehnder interferometer, Nature 422, 415 (2003).

[67] K. T. Law, D. E. Feldman, and Y. Gefen, Electronic Mach-Zehnder interferometer as a tool to probe fractional statistics, Phys. Rev. B 74, 045319 (2006).

[68] P. Bonderson, A. Kitaev, and K. Shtengel, Detecting nonAbelian Statistics in the $v=5 / 2$ Fractional Quantum Hall State, Phys. Rev. Lett. 96, 016803 (2006).

[69] F. E. Camino, W. Zhou, and V. J. Goldman, Realization of a Laughlin quasiparticle interferometer: Observation of fractional statistics, Phys. Rev. B 72, 075342 (2005).

[70] N. Ofek, A. Bid, M. Heiblum, A. Stern, V. Umansky, and D. Mahalu, Role of interactions in an electronic Fabry-Perot interferometer operating in the quantum Hall effect regime, Proc. Natl. Acad. Sci. 107, 5276 (2010).

[71] D. T. McClure, W. Chang, C. M. Marcus, L. N. Pfeiffer, and K. W. West, Fabry-Perot Interferometry with Fractional Charges, Phys. Rev. Lett. 108, 256804 (2012).

[72] R. L. Willett, C. Nayak, K. Shtengel, L. N. Pfeiffer, and K. W. West, Magnetic-Field-Tuned Aharonov-Bohm Oscillations and Evidence for non-Abelian Anyons at $v=5 / 2$, Phys. Rev. Lett. 111, 186401 (2013).

[73] J. Nakamura, S. Fallahi, H. Sahasrabudhe, R. Rahman, S. Liang, G. C. Gardner, and M. J. Manfra, Aharonov-Bohm interference of fractional quantum hall edge modes, Nat. Phys. 15, 563 (2019).

[74] R. L. Willett, K. Shtengel, C. Nayak, L. N. Pfeiffer, Y. J. Chung, M. L. Peabody, K. W. Baldwin, and K. W. West, Interference measurements of non-Abelian e/4 and Abelian e/2 quasiparticle braiding, arXiv:1905.10248 (2019).

[75] H. Bartolomei, M. Kumar, R. Bisognin, A. Marguerite, J.-M. Berroir, E. Bocquillon, B. Plaçais, A. Cavanna, Q. Dong, U. Gennser, Y. Jin, and G. Fève, Fractional statistics in anyon collisions, Science 368, 173 (2020).

[76] C. K. Hong, Z. Y. Ou, and L. Mandel, Measurement of Subpicosecond Time Intervals between two Photons by Interference, Phys. Rev. Lett. 59, 2044 (1987).

[77] A. Zeilinger, Quantum entanglement: A fundamental concept finding its applications, Phys. Scr. T76, 203 (1998).

[78] J. G. Rarity, P. R. Tapster, E. Jakeman, T. Larchuk, R. A. Campos, M. C. Teich, and B. E. A. Saleh, Two-Photon Interference in a Mach-Zehnder Interferometer, Phys. Rev. Lett. 65, 1348 (1990).

[79] M. Michler, K. Mattle, H. Weinfurter, and A. Zeilinger, Interferometric Bell-state analysis, Phys. Rev. A 53, R1209 (1996).

[80] Q. Wang, Y.-S. Zhang, Y.-F. Huang, and G.-C. Guo, Simulating the fourth-order interference phenomenon of anyons with photon pairs, Eur. Phys. J. D 42, 179 (2007).

[81] J. C. F. Matthews, K. Poulios, J. D. A. Meinecke, A. Politi, A. Peruzzo, N. Ismail, K. Wörhoff, M. G. Thompson, and
J. L. O'Brien, Observing fermionic statistics with photons in arbitrary processes, Sci. Rep. 3, 1539 (2013).

[82] A. Lerda and S. Sciuto, Anyons and quantum groups, Nucl. Phys. B 401, 613 (1993).

[83] A. Dasnières de Veigy and S. Ouvry, Equation of State of an Anyon gas in a Strong Magnetic Field, Phys. Rev. Lett. 72, 600 (1994).

[84] S. Ouvry and A. P. Polychronakos, Mapping the calogero model on the anyon model, Nucl. Phys. B. 936, 189 (2018).

[85] S. Ouvry, On the relation between the anyon and calogero models, Phys. Lett. B 510, 335 (2001).

[86] M. D. Girardeau, Anyon-Fermion Mapping and Applications to Ultracold Gases in Tight Waveguides, Phys. Rev. Lett. 97, 100402 (2006).

[87] C. Vitoriano and M. D. Coutinho-Filho, Fractional Statistics and Quantum Scaling Properties of the Hubbard Chain with Bond-Charge Interaction, Phys. Rev. Lett. 102, 146404 (2009).

[88] G. Tang, S. Eggert, and A. Pelster, Ground-state properties of anyons in a one-dimensional lattice, New J. Phys. 17, 123016 (2015).

[89] C. Yannouleas and U. Landman, Anyon optics with timeof-flight two-particle interference of double-well-trapped interacting ultracold atoms, Phys. Rev. A 100, 013605 (2019).

[90] B. Fang, P. Vignolo, M. Gattobigio, C. Miniatura, and A. Minguzzi, Exact solution for the degenerate ground-state manifold of a strongly interacting one-dimensional BoseFermi mixture, Phys. Rev. A 84, 023626 (2011).

[91] A. S. Dehkharghani, F. F. Bellotti, and N. T. Zinner, Analytical and numerical studies of Bose-Fermi mixtures in a one-dimensional harmonic trap, J. Phys. B 50, 144002 (2017).

[92] J. Decamp, J. Jünemann, M. Albert, M. Rizzi, A. Minguzzi, and P. Vignolo, Strongly correlated onedimensional Bose-Fermi quantum mixtures: Symmetry and correlations, New J. Phys. 19, 125001 (2017).

[93] T. Fukuhara, S. Sugawa, Y. Takasu, and Y. Takahashi, Alloptical formation of quantum degenerate mixtures, Phys. Rev. A 79, 021601(R) (2009).

[94] R. Onofrio, Cooling and thermometry of atomic Fermi gases, Phys.-Uspekhi 59, 1129 (2016).

[95] T. Sowiński and M. Á. García-March, One-dimensional mixtures of several ultracold atoms: A review, Rep. Prog. Phys. 82, 104401 (2019).

[96] S. H. Simon, The Oxford Solid State Basics (Oxford University Press, Oxford, 2013).

[97] H. B. Callen, Thermodynamics and an Introduction to Thermostatistics (Wiley, New York, 1985).

[98] F. L. Curzon and B. Ahlborn, Efficiency of a carnot engine at maximum power output, Am. J. Phys. 43, 22 (1975).

[99] M. H. Rubin, Optimal configuration of a class of irreversible heat engines. i, Phys. Rev. A 19, 1272 (1979).

[100] K. H. Hoffmann, J. M. Burzler, and S. Schubert, Endoreversible thermodynamics, J. Non-Equilib. Thermodyn. 22, 311 (1997).

[101] T. Feldmann, E. Geva, R. Kosloff, and P. Salamon, Heat engines in finite time governed by master equations, Am. J. Phys. 64, 485 (1996). 
[102] Y. Rezek and R. Kosloff, Irreversible performance of a quantum harmonic heat engine, New J. Phys. 8, 83 (2006).

[103] T. Feldmann and R. Kosloff, Short time cycles of purely quantum refrigerators, Phys. Rev. E 85, 051114 (2012).

[104] A. d. Campo, J. Goold, and M. Paternostro, More bang for your buck: Super-adiabatic quantum engines, Sci. Rep. 4, 6208 (2014).

[105] M. Beau, J. Jaramillo, and A. del Campo, Scaling-up quantum heat engines efficiently via shortcuts to adiabaticity, Entropy 18, 168 (2016).

[106] Y. Zheng, P. Hänggi, and D. Poletti, Occurrence of discontinuities in the performance of finite-time quantum Otto cycles, Phys. Rev. E 94, 012137 (2016).

[107] O. Abah and E. Lutz, Energy efficient quantum machines, Europhys. Lett. 118, 40005 (2017).

[108] O. Abah and E. Lutz, Performance of shortcut-toadiabaticity quantum engines, Phys. Rev. E 98, 032121 (2018).

[109] J.-F. Chen, C.-P. Sun, and H. Dong, Boosting the performance of quantum Otto heat engines, Phys. Rev. E 100, 032144 (2019).

[110] J.-F. Chen, C.-P. Sun, and H. Dong, Achieve higher efficiency at maximum power with finite-time quantum Otto cycle, Phys. Rev. E 100, 062140 (2019).

[111] M. V. S. Bonança, Approaching carnot efficiency at maximum power in linear response regime, J. Stat. Mech.: The. Exp. 2019, 123203 (2019).

[112] O. Abah and M. Paternostro, Shortcut-to-adiabaticity Otto engine: A twist to finite-time thermodynamics, Phys. Rev. E 99, 022110 (2019).

[113] K. Funo, N. Lambert, B. Karimi, J. P. Pekola, Y. Masuyama, and F. Nori, Speeding up a quantum refrigerator via counterdiabatic driving, Phys. Rev. B 100, 035407 (2019).

[114] S. Lee, M. Ha, J.-M. Park, and H. Jeong, Finite-time quantum Otto engine: Surpassing the quasistatic efficiency due to friction, Phys. Rev. E 101, 022127 (2020).

[115] R. Uzdin and R. Kosloff, Universal features in the efficiency at maximal work of hot quantum Otto engines, Europhys. Lett. 108, 40001 (2014).

[116] F. J. Peña and E. Muñoz, Magnetostrain-driven quantum engine on a graphene flake, Phys. Rev. E 91, 052152 (2015).

[117] K. Zhang, F. Bariani, and P. Meystre, Quantum Optomechanical Heat Engine, Phys. Rev. Lett. 112, 150602 (2014).

[118] Y. Zheng and D. Poletti, Quantum statistics and the performance of engine cycles, Phys. Rev. E 92, 012110 (2015).

[119] J. Jaramillo, M. Beau, and A. del Campo, Quantum supremacy of many-particle thermal machines, New J. Phys. 18, 075019 (2016).

[120] F. Peña, A. González, A. Nunez, P. Orellana, R. Rojas, and P. Vargas, Magnetic engine for the single-particle landau problem, Entropy 19, 639 (2017).

[121] X. L. Huang, D. Y. Guo, S. L. Wu, and X. X. Yi, Multilevel quantum Otto heat engines with identical particles, Quantum Inf. Process. 17, 27 (2017).

[122] S. Deffner, Efficiency of harmonic quantum Otto engines at maximal power, Entropy 20, 875 (2018).
[123] J. Li, T. Fogarty, S. Campbell, X. Chen, and T. Busch, An efficient nonlinear feshbach engine, New J. Phys. 20, 015005 (2018).

[124] M. Kloc, P. Cejnar, and G. Schaller, Collective performance of a finite-time quantum Otto cycle, Phys. Rev. E 100, 042126 (2019).

[125] N. Yunger Halpern, C. D. White, S. Gopalakrishnan, and G. Refael, Quantum engine based on many-body localization, Phys. Rev. B 99, 024203 (2019).

[126] F. J. Peña, O. Negrete, G. Alvarado Barrios, D. Zambrano, A. González, A. S. Nunez, P. A. Orellana, and P. Vargas, Magnetic Otto engine for an electron in a quantum dot: Classical and quantum approach, Entropy 21, 512 (2019).

[127] N. M. Myers and S. Deffner, Bosons outperform fermions: The thermodynamic advantage of symmetry, Phys. Rev. E 101, 012110 (2020).

[128] G. Watanabe, B. P. Venkatesh, P. Talkner, M.-J. Hwang, and A. del Campo, Quantum Statistical Enhancement of the Collective Performance of Multiple Bosonic Engines, Phys. Rev. Lett. 124, 210603 (2020).

[129] F. J. Peña, D. Zambrano, O. Negrete, G. De Chiara, P. A. Orellana, and P. Vargas, Quasistatic and quantumadiabatic Otto engine for a two-dimensional material: The case of a graphene quantum dot, Phys. Rev. E 101, 012116 (2020).

[130] Z. Smith, P. S. Pal, and S. Deffner, Endoreversible Otto engines at maximal power, J. Non-Equilib. Thermodyn. 45, 305 (2020).

[131] R. Kosloff, A quantum mechanical open system as a model of a heat engine, J. Chem. Phys. 80, 1625 (1984).

[132] O. Abah, J. Roßnagel, G. Jacob, S. Deffner, F. SchmidtKaler, K. Singer, and E. Lutz, Single-Ion Heat Engine at Maximum Power, Phys. Rev. Lett. 109, 203006 (2012).

[133] K. Husimi, Miscellanea in elementary quantum mechanics, II, Prog. Theor. Phys. 9, 381 (1953).

[134] E. Torrontegui, S. Ibáñez, S. Martínez-Garaot, M. Modugno, A. del Campo, D. Guéry-Odelin, A. Ruschhaupt, X. Chen, and J. G. Muga, Shortcuts to adiabaticity, Adv. At. Mol. Opt. Phys. 62, 117 (2013).

[135] M. Demirplak and S. A. Rice, Adiabatic population transfer with control fields, J. Phys. Chem. A 107, 9937 (2003).

[136] M. V. Berry, Transitionless quantum driving, J. Phys. A: Math. Theor. 42, 365303 (2009).

[137] S. Deffner, C. Jarzynski, and A. del Campo, Classical and Quantum Shortcuts to Adiabaticity for Scale-Invariant Driving, Phys. Rev. X 4, 021013 (2014).

[138] S. Deng, A. Chenu, P. Diao, F. Li, S. Yu, I. Coulamy, A. del Campo, and $\mathrm{H}$. Wu, Superadiabatic quantum friction suppression in finite-time thermodynamics, Sci. Adv. 4, eaar5909 (2018).

[139] X. Chen, A. Ruschhaupt, S. Schmidt, A. del Campo, D. Guéry-Odelin, and J. G. Muga, Fast Optimal Frictionless Atom Cooling in Harmonic Traps: Shortcut to Adiabaticity, Phys. Rev. Lett. 104, 063002 (2010).

[140] A. d. Campo and M. G. Boshier, Shortcuts to adiabaticity in a time-dependent box, Sci. Rep. 2, 648 (2012).

[141] S. Masuda and K. Nakamura, Fast-forward of adiabatic dynamics in quantum mechanics, Proc. R. Soc. A. 466, 1135 (2009). 
[142] S. Masuda and K. Nakamura, Acceleration of adiabatic quantum dynamics in electromagnetic fields, Phys. Rev. A 84, 043434 (2011).

[143] E. Torrontegui, S. Martínez-Garaot, A. Ruschhaupt, and J. G. Muga, Shortcuts to adiabaticity: Fast-forward approach, Phys. Rev. A 86, 013601 (2012).

[144] S. Masuda, K. Nakamura, and A. del Campo, HighFidelity Rapid Ground-State Loading of an Ultracold Gas Into an Optical Lattice, Phys. Rev. Lett. 113, 063003 (2014).

[145] S. Deffner, Shortcuts to adiabaticity: Suppression of pair production in driven Dirac dynamics, New J. Phys. 18, 012001 (2015).

[146] D. Stefanatos, Optimal shortcuts to adiabaticity for a quantum piston, Automatica 49, 3079 (2013).

[147] T. V. Acconcia, M. V. S. Bonança, and S. Deffner, Shortcuts to adiabaticity from linear response theory, Phys. Rev. E 92, 042148 (2015).

[148] S. Campbell, G. De Chiara, M. Paternostro, G. M. Palma, and R. Fazio, Shortcut to Adiabaticity in the LipkinMeshkov-Glick Model, Phys. Rev. Lett. 114, 177206 (2015).

[149] Y. Rosales-Cabara, G. Manfredi, G. Schnoering, P.-A. Hervieux, L. Mertz, and C. Genet, Optimal protocols and universal time-energy bound in brownian thermodynamics, Phys. Rev. Res. 2, 012012(R) (2020).

[150] B. d. L. Bernardo, Time-rescaled quantum dynamics as a shortcut to adiabaticity, Phys. Rev. Res. 2, 013133 (2020).

[151] A. Roychowdhury and S. Deffner, Time-rescaling of Dirac dynamics: Shortcuts to adiabaticity in ion traps and Weyl semimetals, Entropy 23, 81 (2021).

[152] A. Patra and C. Jarzynski, Shortcuts to adiabaticity using flow fields, New J. Phys. 19, 125009 (2017).

[153] C. Jarzynski, S. Deffner, A. Patra, and Y. Subasi, Fast forward to the classical adiabatic invariant, Phys. Rev. E 95, 032122 (2017).
[154] H. R. Lewis and P. G. L. Leach, A direct approach to finding exact invariants for one-dimensional timedependent classical hamiltonians, J. Math. Phys. 23, 2371 (1982).

[155] Z. Holmes, J. Anders, and F. Mintert, Enhanced Energy Transfer to an Optomechanical Piston from Indistinguishable Photons, Phys. Rev. Lett. 124, 210601 (2020).

[156] Y. Chen, C. Lee, L. Lu, D. Liu, Y.-K. Wu, L.-T. Feng, M. Li, C. Rockstuhl, G.-P. Guo, G.-C. Guo, M. Tame, and X.-F. Ren, Quantum plasmonic N00N state in a silver nanowire and its use for quantum sensing, Optica 5, 1229 (2018).

[157] B. Yadin, B. Morris, and G. Adesso, Extracting work from mixing indistinguishable systems: A quantum Gibbs "paradox", arXiv:2006.12482[quant-ph] (2020).

[158] M. Lebugle, M. Gräfe, R. Heilmann, A. Perez-Leija, S. Nolte, and A. Szameit, Experimental observation of n00n state bloch oscillations, Nat. Commun. 6, 8273 (2015).

[159] B. Paredes, P. Fedichev, J. I. Cirac, and P. Zoller, $\frac{1}{2}$-anyons in Small Atomic Bose-Einstein Condensates, Phys. Rev. Lett. 87, 010402 (2001).

[160] M. V. Medvedev, Properties of Particles Obeying Ambiguous Statistics, Phys. Rev. Lett. 78, 4147 (1997).

[161] F. Lange, S. Ejima, and H. Fehske, Anyonic Haldane Insulator in one Dimension, Phys. Rev. Lett. 118, 120401 (2017).

[162] J. Sicks and H. Rieger, Haldane insulator in the 1d nearestneighbor extended bose-hubbard model with cavitymediated long-range interactions, Eur. Phys. J. B 93, 104 (2020).

[163] J. Majhi, S. Ghosh, and S. K. Maiti, Relativistic Anyon Beam: Construction and Properties, Phys. Rev. Lett. 123, 164801 (2019).

[164] A. Strominger, Black Hole Statistics, Phys. Rev. Lett. 71, 3397 (1993). 